\title{
Sugar Sweetened Beverages Intake and Risk of Non-Communicable Chronic Diseases in Longitudinal Studies: A Systematic Review and Meta-Analysis with 1.5 Million Individuals
}

\author{
Leonardo Pozza Santos ( $\square$ leonardo_pozza@yahoo.com.br) \\ Universidade Federal de Pelotas https://orcid.org/0000-0002-3993-3786 \\ Denise Petrucci Gigante \\ Federal University of Pelotas: Universidade Federal de Pelotas \\ Felipe Mendes Delpino \\ Federal University of Pelotas: Universidade Federal de Pelotas \\ Ana Paula Maciel \\ Federal University of Pelotas: Universidade Federal de Pelotas \\ Renata Moraes Bielemann \\ Federal University of Pelotas: Universidade Federal de Pelotas
}

\section{Research Article}

Keywords: Sweetened drinks, Non-infectious diseases, Prospective studies, Review literature

Posted Date: December 2nd, 2021

DOl: https://doi.org/10.21203/rs.3.rs-950662/v1

License: (a) (i) This work is licensed under a Creative Commons Attribution 4.0 International License. Read Full License 


\section{Abstract}

This study aimed to examine longitudinal associations between sugar-sweetened beverages (SSB) intake and type 2 diabetes, obesity, coronary heart disease and stroke in adults. We performed a systematic review and meta-analysis searching for articles in the Pubmed, Lilacs, Web of Science, Cochrane, Embase, and Scopus databases. After screening of titles and abstracts, 27 longitudinal studies were included for the narrative synthesis with all of them presenting medium or high methodological quality. None of the selected studies were from low-income countries and only three were conducted in middle-income countries. Type 2 diabetes was the most investigated disease - outcome in 15 out of 27 studies. Around $80 \%$ of the studies enrolled more than 10,000 individuals in the sample, and almost half of them followed the subjects for less than 10 years. A total of 1.5 million individuals were included in the pooled analyses, and results indicated that SSB intake increased the risk of type 2 diabetes $(R R=1.20 ; 95 \%$ C.I. $1.13-1.28)$, obesity $(R R=1.17 ; 95 \%$ C.I. $1.10-$ 1.25), coronary heart disease $(R R=1.15 ; 95 \%$ C.I. $1.06-1.25)$, and stroke $(R R=1.10 ; 95 \%$ C.I. $1.01-1.19)$ in adults after adjustment for all potential confounders. Our systematic review and meta-analysis demonstrated that consumption of SSB intake appears to increase the risk non-communicable chronic disease, being the strongest evidence for type 2 diabetes. Actions are needed to be taken to reduce the SSB intake and its consequences worldwide.

\section{Introduction}

Non-communicable chronic diseases (NCD) are the leading cause of deaths worldwide [1]-[3], and behavioral risk factors, like unhealthy eating habits, are the main determinants for the development of NCD [4], [5]. Despite the adherence of healthy dietary patterns have recently improved, mainly in high-income countries [6], the consumption of unhealthy food items is still high [6]-[8]. In this context, sugarsweetened beverages (SSB) are among the unhealthy food items most associated with adverse outcomes, especially with NCD [9]-[12]. SSB are beverages sweetened with different forms of added sugar, including carbonated and non-carbonated soft drinks, fruits and sports drinks, and they are characterized by low nutritional quality [13].

In 2010, almost 200 thousand deaths around the world were attributable to SSB consumption, being type 2 diabetes the leading cause [14]. Singh et al. [14] also estimated that the majority of mortality attributable to SSB intake occurred in low- and middle-income countries (LMIC), while high-income countries accounted for a quarter of the total deaths. Since then, the consumption of SSB is apparently decreasing, although the prevalence remains high, mainly in specific sub-groups such as men and younger individuals [15]-[19].

Association between SSB intake and health-related outcomes is well reported in literature. Several investigations have shown that high SSB intake increases the risk of obesity-related diseases [9], [10], [12], [20]. However, the effects of SSB consumption on NCD, like type 2 diabetes, appear to be only partially explained by obesity status, being part of this effect independent of adiposity [11]. Despite the scientific literature regarding the association between SSB consumption and NCD has increased in the last years, the magnitude of the association is quite heterogeneous among studies.

As SSB intake is an important and suitable target for environmental interventions [20], understanding the strength, consistency and biological gradient of the association between SSB consumption and health-related outcomes is important. Based on these evidence, comprehensive approaches to reduce SSB consumption, such as public health interventions and taxation, may be better planned. Therefore, we performed a systematic review and meta-analysis of longitudinal studies with adults to assess the association between SSB intake and NCD, namely type 2 diabetes, obesity, coronary heart disease (CHD) and stroke.

\section{Methods}

\section{Search strategy}

This study is a systematic review and meta-analysis performed according to the PRISMA statement [21]. We searched studies that observed the association between SSB intake and type 2 diabetes, obesity, CHD or stroke in adults published until December 2020. We considered SSB as any drink with added sugar, such as soft drinks, artificial fruit juices, and sports drinks.

The articles' search was carried out using the Pubmed, Lilacs, Web of Science, Cochrane, Embase, and Scopus databases. The terms used on the articles' search were based on the MeSH terms along with other non-MeSH terms to complement the searching, as following: "soft drinks", "processed juice", "sugar-sweetened drinks", "sugar-sweetened beverage", "fruit juices", "artificially-sweetened soda", "artificially sweetened beverages", "sugar-sweetened soft drinks", "diet soda", "fruit-flavored drinks", "sports and energy drinks", "bottled fruit juices" with the Boolean operator "OR" between the terms, and "AND" "obesity", "coronary disease", "diabetes", "cerebral vascular 
accident", "cerebrovascular accident", "stroke" and "cerebrovascular disease", also utilizing "OR" between the terms. This systematic review was registered and approved on the International Prospective Register of Systematic Reviews (PROSPERO) (CRD420212342060). Inclusion criteria

We included longitudinal studies (cohort) carried out with adults ( $\geq 20$ years old) not belonging to specific groups (individuals with an initial diagnosis of any morbidity or institutionalized, for example) and investigating the association between the intake of one or more types of SSB with at least one of the four outcomes of interest (type 2 diabetes, obesity, CHD and stroke).

\section{Exclusion criteria}

Systematic and non-systematic reviews, cross-sectional and intervention studies, and studies with animals or in vitro were excluded. We also excluded those investigations that reported data from dietary patterns without showing results exclusively for sugar sweetened beverages.

\section{Study selection}

Two independent reviewers (APM and FMD) conducted the articles' selection, and, in case of disagreements, the judgment was left to a third one (DPG). After excluding duplicates, the selection started by reading the titles, followed by abstracts, according to the inclusion and exclusion criteria. Finally, the eligible articles were selected from a full reading of the manuscripts. We also evaluated the reference lists of all included articles to identify relevant studies that might not have been initially located.

\section{Meta-analysis}

In the meta-analysis, we included studies that evaluated the association between SSB intake and risk of type 2 diabetes, obesity, CHD and/or stroke, and reported data as Odds Ratio (OR), Hazard Ratio (HR) or Relative Risk (RR). We pooled the RR across studies through the DerSimonian and Laird random-effects model [22]. We considered HR values as RR since their results are very similar [23]. ORs were converted into RRs using the following equation: $R R=O R /\left[\left(1-P_{0}\right)+\left(P_{0} X O R\right)\right]$, in which Po represents the incidence of the outcome of interest in the nonexposed group [24]. We used the fixed-effects model to calculate article-specific RR for studies that assessed values stratified by sex or different subgroups. In case of missing information for OR, HR or RR in the manuscripts, we contacted authors in two different occasions, with a delay from 30 days between each contact.

For studies assessing the isolated risk of different types of SSB, we included results for the most consumed beverage in the sample under analysis. For all studies, the cut-off point assumed was one or more servings of SSB per day. We considered low/moderate intake one or less portion of SSB per day and the intake of two or more portions of SSB per day was classified as high intake. The HR, OR and RR with the greatest adjustments for confounding were considered as the final effect.

We used the Higgins $\mathrm{I}^{2}$ test to estimate statistical heterogeneity among studies [25]. This test varies from $0-100 \%$ and the higher the values the higher heterogeneity. We used the cut-off points proposed by Higgins et al. [26] to classify heterogeneity, considering moderate heterogeneity those values of $\mathrm{I}^{2}$ between $50 \%$ and $74.9 \%$ and high heterogeneity when $\mathrm{I}^{2}$ values were $\geq 75 \%$. Analyses were performed through $\mathrm{R}$ language utilizing the Rstudio program and miniMeta package [27].

\section{Quality of evidence and risk of publication bias}

The methodological quality of the studies was assessed by two independent reviewers (APM and FMD), using the Newcastle-Ottawa Scale (NOS), which assesses longitudinal studies quality based on sample selection criteria, comparability between groups, and evaluation of exposure or outcome [28]. This scale includes eight items, and each of them receives a star when the study is classified as high quality, except for the comparability item, which can receive two stars when results were adjusted by sex and other factors. Thus, the total NOS scale score varied from 0 to 9 . We classified studies which scored less than five points in the NOS with poor quality, those studies with five or six points as medium quality, while articles which scored seven or more points were classified as high-quality studies. Such classification was based on previous published papers [29], [30].

Risk of publication bias was assessed using the funnel plot, and the funnel plot asymmetry was tested using the Egger's and Begg's test [31].

\section{Results}


Figure 1 summarizes the studies selection process. A total of 18755 titles and abstracts were screened after duplicates have been removed. The screening of titles and abstracts retrieved 98 articles for further consideration. After exclusion of 69 manuscripts which have not met the inclusion criteria, our systematic review included findings from 27 longitudinal studies evaluating the association between SSB intake and type 2 diabetes, obesity, CHD and stroke (Figure 1).

Notwithstanding the 27 included studies have been conducted in 12 different countries, more than a half of them came from United States. In addition, almost all studies were conducted in high-income countries, with only three of them from middle-income countries (China, Thailand, and Mexico). Almost half of the included studies enrolled between 10,000 and 50,000 individuals in the sample. In terms of follow-up period, almost $20 \%$ of the selected papers followed their sample for more than 20 years. Finally, type 2 diabetes was the most frequent outcome in the included studies $(n=15)$. Stroke was the outcome in five, while obesity was the outcome in four studies and $\mathrm{CHD}$ in three of them (Table 1). 
Table 1

Characteristics of the 27 papers selected by systematic review on the longitudinal association between sugar sweetened beverages intake and noncommunicable chronic diseases in adults.

\begin{tabular}{|c|c|c|}
\hline Category & Number of studies & $\%$ \\
\hline \multicolumn{3}{|l|}{ Year of publication } \\
\hline Between 2000 and 2010 & 7 & 25.9 \\
\hline Between 2010 and 2020 & 20 & 74.1 \\
\hline \multicolumn{3}{|l|}{ Region } \\
\hline North America & 15 & 55.6 \\
\hline Europe and Central Asia & 6 & 22.2 \\
\hline South Asia or East Asia and Pacific & 5 & 18.5 \\
\hline Latin America and Caribbean & 1 & 3.7 \\
\hline \multicolumn{3}{|l|}{ Country's income level } \\
\hline High-income & 24 & 88.9 \\
\hline Middle-income & 3 & 11.1 \\
\hline \multicolumn{3}{|l|}{ Gender } \\
\hline Only male & 2 & 7.4 \\
\hline Only female & 7 & 25.9 \\
\hline Both & 18 & 66.7 \\
\hline \multicolumn{3}{|l|}{ Sample size } \\
\hline$<10,000$ & 5 & 18.5 \\
\hline $10,000-50,000$ & 13 & 48.2 \\
\hline$>50,000$ & 9 & 33.3 \\
\hline \multicolumn{3}{|l|}{ Follow-up duration } \\
\hline Up to 10 years & 13 & 48.2 \\
\hline 10 to 20 years & 9 & 33.3 \\
\hline More than 20 years & 5 & 18.5 \\
\hline \multicolumn{3}{|l|}{ Outcome } \\
\hline Type 2 diabetes & 15 & 55.6 \\
\hline Obesity & 4 & 14.8 \\
\hline Coronary heart diseases & 3 & 11.1 \\
\hline Stroke & 5 & 18.5 \\
\hline \multicolumn{3}{|c|}{ Quality assessment (Newcastle-Ottawa Scale) } \\
\hline Medium & 6 & 22.2 \\
\hline High & 21 & 77.8 \\
\hline Total & 27 & 100.0 \\
\hline
\end{tabular}

\section{Quality assessment}


The scores obtained in the NOS varied from 5 to 9 . The majority of articles were classified as high-quality studies (21 studies; $77.8 \%$ ), while six were classified as medium quality. None of the included articles scored less than five points in the NOS. Five studies classified as medium quality did not clearly state about the representativeness of the exposed cohort, five studies did not report on the assessment of outcome and four medium quality studies did not state well the adequacy of follow-up cohorts (Supplementary table 1).

\section{Association between SSB intake and type 2 diabetes}

Fifteen investigations assessed the association between SSB intake and diabetes (Table 2). In 13 studies, diabetes was the only outcome [9], [32]-[43], while one study assessed diabetes and weight gain as the main outcomes [44] and another one assessed diabetes and stroke [45]. Results were considerably consistent in showing that SSB intake has been associated with higher risk of type 2 diabetes, since only one study did not find any statistically significant association [32]. Schultze et al. [44], Palmer et al. [33], de Koning et al. [35] and Hirahatake et al. [41] showed that the consumption of more than one portion of SSB per day increased the risk of type 2 diabetes in United States, after controlling for several confounders, including dietary intake and body mass index. In addition, study conducted by the InterAct consortium from eight European countries also revealed positive effects of SSB intake on the risk of type 2 diabetes after adjustment for demographic and lifestyle characteristics [9]. 
Table 2

Description of studies selected by systematic review about the longitudinal effects of sugar sweetened beverages intake on diabetes in

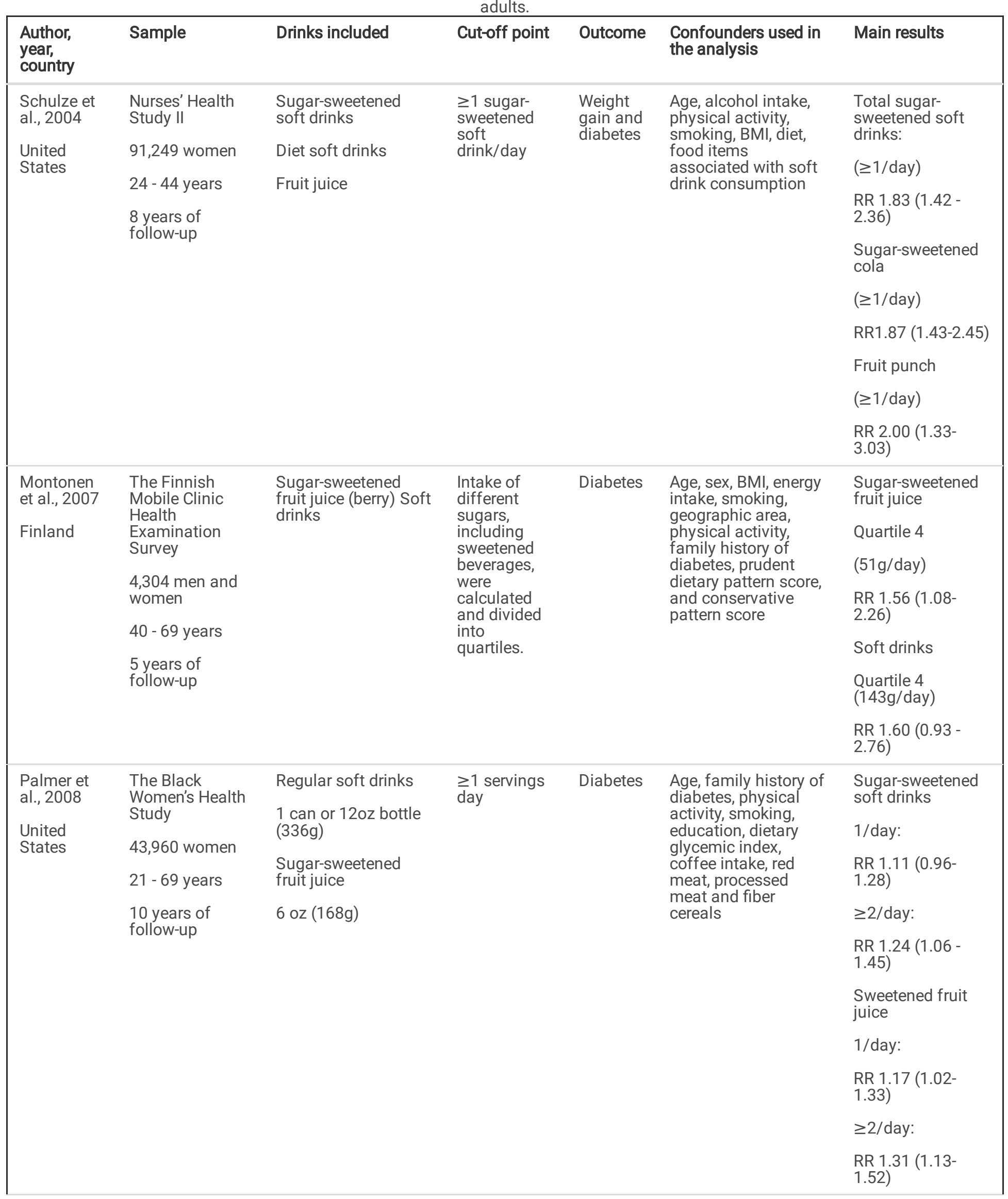




\begin{tabular}{|c|c|c|c|c|c|c|}
\hline $\begin{array}{l}\text { Author, } \\
\text { year, } \\
\text { country }\end{array}$ & Sample & Drinks included & Cut-off point & Outcome & $\begin{array}{l}\text { Confounders used in } \\
\text { the analysis }\end{array}$ & Main results \\
\hline $\begin{array}{l}\text { Nettleton et } \\
\text { al., } 2009 \\
\text { United } \\
\text { States }\end{array}$ & $\begin{array}{l}\text { Multi-Ethnic } \\
\text { Study of } \\
\text { Atherosclerosis } \\
\text { (MESA) } \\
5,011 \text { men and } \\
\text { women } \\
45 \text { - } 84 \text { years } \\
6 \text { years of } \\
\text { follow-up }\end{array}$ & $\begin{array}{l}\text { Diet soft drinks } \\
\text { (diet soft drinks and } \\
\text { unsweetened mineral } \\
\\
\text { water) } \\
\text { Sugar-sweetened } \\
\text { soft drinks (sugar- } \\
\text { sweetened soft } \\
\text { drinks, non-alcoholic } \\
\text { beer and sweetened } \\
\text { mineral water) }\end{array}$ & $\begin{array}{l}\geq 1 \\
\text { serving/day }\end{array}$ & Diabetes & $\begin{array}{l}\text { Age, sex, race, } \\
\text { examination site, } \\
\text { energy intake, } \\
\text { education, time spent } \\
\text { in active and inactive } \\
\text { activities during } \\
\text { leisure, smoking, } \\
\text { regular use of dietary } \\
\text { supplements, intake } \\
\text { of various foods and } \\
\text { nutrients, baseline } \\
\text { waist circumference, } \\
\text { baseline BMI and } \\
\text { change in waist } \\
\text { circumference or } \\
\text { body weight }\end{array}$ & $\begin{array}{l}\text { There was no } \\
\text { association } \\
\text { between } \\
\text { sweetened } \\
\text { beverages and } \\
\text { incidence of } \\
\text { diabetes (data not } \\
\text { shown) }\end{array}$ \\
\hline $\begin{array}{l}\text { Odegaard } \\
\text { et al., } 2010 \\
\text { China }\end{array}$ & $\begin{array}{l}\text { Singapore } \\
\text { Chinese Health } \\
\text { Study } \\
63,256 \\
\text { individuals aged } \\
45-74 \text { years }\end{array}$ & $\begin{array}{l}\text { With sugar: } \\
\text { carbonated soft } \\
\text { drinks, carbonated } \\
\text { drinks } \\
\text { Diet: Low-calorie or } \\
\text { no-calorie soft drinks } \\
\text { and other non-calorie } \\
\text { beverages }\end{array}$ & $\begin{array}{l}\geq 2 \text { servings } \\
\text { / week }\end{array}$ & Diabetes & $\begin{array}{l}\text { Age, sex, height, } \\
\text { physical activity, } \\
\text { smoking, education, } \\
\text { nutritional intake, and } \\
\text { adiposity measures }\end{array}$ & $\begin{array}{l}\text { Soft drinks ( } \geq 2 \\
\text { per week) } \\
\text { RR 1.34 (1.17 - } \\
1.52)\end{array}$ \\
\hline $\begin{array}{l}\text { de Koning } \\
\text { et al., } 2011 \\
\text { United } \\
\text { States }\end{array}$ & $\begin{array}{l}\text { Health } \\
\text { Professionals } \\
\text { Follow-Up Study } \\
\text { (HPFS) } \\
40,389 \text { men } \\
40-75 \text { years } \\
20 \text { years of } \\
\text { follow-up }\end{array}$ & $\begin{array}{l}\text { Caffeinated and } \\
\text { caffeine-free soft } \\
\text { drinks, other sugar- } \\
\text { sweetened } \\
\text { carbonated } \\
\text { beverages and sugar- } \\
\text { sweetened non- } \\
\text { carbonated } \\
\text { beverages } \\
\text { Artificially } \\
\text { sweetened, } \\
\text { caffeinated/caffeine- } \\
\text { free and non- } \\
\text { carbonated low- } \\
\text { calorie beverages } \\
\text { Serving: } \\
\text { Quartile } 1 \text { (never) } \\
\text { Quartile } 2 \text { (2/month) } \\
\text { Quartile } 3 \text { (1-4 } \\
\text { times/week) } \\
\text { Quartile } 4 \text { (4.5 } \\
\text { times/week to } 7.5 \\
\text { times/day) }\end{array}$ & $\begin{array}{l}1 \\
\text { serving/day }\end{array}$ & Diabetes & $\begin{array}{l}\text { Smoking, physical } \\
\text { activity, alcohol } \\
\text { intake, family history } \\
\text { of type } 2 \text { diabetes, } \\
\text { high blood pressure, } \\
\text { use of diuretics, } \\
\text { multivitamin intake, } \\
\text { diet quality, weight } \\
\text { change and } \\
\text { adherence to a low- } \\
\text { calorie diet, total } \\
\text { energy intake and } \\
\text { BMI }\end{array}$ & $\begin{array}{l}\text { Total sugar- } \\
\text { sweetened } \\
\text { beverages } \\
(1 / \text { day) } \\
\text { HR } 1.16(1.08 \text { - } \\
1.25)\end{array}$ \\
\hline
\end{tabular}

RR - Relative risk; HR - Hazard ratio; OR - Odds ratio; BMI - Body Mass Index; CHD - Coronary heart disease. 


\begin{tabular}{|c|c|c|c|c|c|c|}
\hline $\begin{array}{l}\text { Author, } \\
\text { year, } \\
\text { country }\end{array}$ & Sample & Drinks included & Cut-off point & Outcome & $\begin{array}{l}\text { Confounders used in } \\
\text { the analysis }\end{array}$ & Main results \\
\hline $\begin{array}{l}\text { Eshak et } \\
\text { al., } 2013 \\
\text { Japan }\end{array}$ & $\begin{array}{l}\text { The Japan } \\
\text { Public Health } \\
\text { Center-based } \\
\text { (JPHC) } \\
27,585 \text { men and } \\
\text { women } \\
40 \text { - } 59 \text { years } \\
10 \text { years of } \\
\text { follow-up }\end{array}$ & $\begin{array}{l}\text { Soft drinks (cola, } \\
\text { flavored juices and } \\
\text { non- } 100 \% \text { fruit) } \\
100 \% \text { fruit juice } \\
100 \% \text { vegetable juice } \\
1 \text { glass }(250 \mathrm{ml})\end{array}$ & $\begin{array}{l}\text { Drinking } \\
\text { almost every } \\
\text { day } \\
\text { ( } \geq 1 \\
\text { serving/day) }\end{array}$ & Diabetes & $\begin{array}{l}\text { Age, BMI, family } \\
\text { history of diabetes, } \\
\text { education, } \\
\text { occupation, smoking, } \\
\text { alcohol intake, history } \\
\text { of hypertension, } \\
\text { leisure-time physical } \\
\text { activity, coffee } \\
\text { consumption and } \\
\text { green tea intake, } \\
\text { intake of some } \\
\text { nutrients and total } \\
\text { energy intake }\end{array}$ & $\begin{array}{l}\text { Drinking soda } \\
\text { almost every day } \\
\text { OR } 1.79(1.11 \text { - } \\
2.89) \text { women } \\
\text { OR } 0.98 \text { (0.68 - } \\
\text { 1.42) men }\end{array}$ \\
\hline $\begin{array}{l}\text { Fagherazzi } \\
\text { et al., } 2013 \\
\text { France }\end{array}$ & $\begin{array}{l}\text { Etude } \\
\text { Epidémiologique } \\
\text { auprès des } \\
\text { femmes de la } \\
\text { Mutuelle } \\
\text { Générale de } \\
\text { l'Education } \\
\text { Nationale- } \\
\text { European } \\
\text { Prospective } \\
\text { Investigation } \\
\text { into Cancer and } \\
\text { Nutrition cohort } \\
\text { (E3N Study) } \\
66,118 \text { women } \\
14 \text { years of } \\
\text { follow-up }\end{array}$ & $\begin{array}{l}100 \% \text { fruit juices } \\
\text { Sugary drinks (sugar- } \\
\text { sweetened/artificially } \\
\text { sweetened soft } \\
\text { drinks or water with } \\
\text { added fruit syrup) }\end{array}$ & $\begin{array}{l}>359 \\
\mathrm{~mL} / \text { week of } \\
\text { sugar- } \\
\text { sweetened } \\
\text { beverages }\end{array}$ & Diabetes & $\begin{array}{l}\text { Education, smoking, } \\
\text { physical activity, } \\
\text { hypertension, } \\
\text { hypercholesterolemia, } \\
\text { use of hormone } \\
\text { replacement therapy, } \\
\text { family history of } \\
\text { diabetes, self- } \\
\text { reported use of } \\
\text { antidiabetic drugs, } \\
\text { alcohol intake, intake } \\
\text { of omega-3 fatty } \\
\text { acids, intake of } \\
\text { carbohydrates, } \\
\text { coffee, fruits, } \\
\text { vegetables and } \\
\text { consumption of } \\
\text { processed meats, } \\
\text { total energy intake } \\
\text { and Mediterranean } \\
\text { dietary pattern }\end{array}$ & $\begin{array}{l}>359 \mathrm{~mL} / \text { week o } \\
\text { sugar-sweetened } \\
\text { beverages } \\
\text { HR } 1.30(1.02 \text { - } \\
1.66)\end{array}$ \\
\hline $\begin{array}{l}\text { Romaguera } \\
\text { et al., } 2013 \\
\text { Europe }\end{array}$ & $\begin{array}{l}\text { European } \\
\text { Prospective } \\
\text { Investigation on } \\
\text { Cancer and } \\
\text { Nutrition (EPIC- } \\
\text { InterAct) } \\
15,374 \text { men and } \\
\text { women } \\
16 \text { years of } \\
\text { follow-up }\end{array}$ & $\begin{array}{l}\text { Soft drinks, } \\
\text { carbonated drinks, } \\
\text { isotonic drinks and } \\
\text { diluted syrup } \\
\text { Juices and nectars } \\
1 \text { glass } 8.8 \text { oz }(250 \mathrm{~g})\end{array}$ & $\begin{array}{l}\geq 1 \\
\text { glass/day }\end{array}$ & Diabetes & $\begin{array}{l}\text { Sex, smoking, } \\
\text { alcohol, } \\
\text { consumption, } \\
\text { education and } \\
\text { physical activity, total } \\
\text { juices, nectars and } \\
\text { soft drinks were } \\
\text { mutually adjusted, } \\
\text { total energy intake, } \\
\text { BMl, waist } \\
\text { circumference, } \\
\text { presence of } \\
\text { hyperlipidemia and } \\
\text { hypercholesterolemia, } \\
\text { consumption of } \\
\text { various food items } \\
\text { and Mediterranean } \\
\text { diet index }\end{array}$ & $\begin{array}{l}\text { Total sugar- } \\
\text { sweetened soft } \\
\text { drinks } \\
(\geq 1 \text { glass/day) } \\
\text { HR } 1.21 \text { (1.05, } \\
1.41)\end{array}$ \\
\hline
\end{tabular}

RR - Relative risk; HR - Hazard ratio; OR - Odds ratio; BMI - Body Mass Index; CHD - Coronary heart disease. 


\begin{tabular}{|c|c|c|c|c|c|c|}
\hline $\begin{array}{l}\text { Author, } \\
\text { year, } \\
\text { country }\end{array}$ & Sample & Drinks included & Cut-off point & Outcome & $\begin{array}{l}\text { Confounders used in } \\
\text { the analysis }\end{array}$ & Main results \\
\hline $\begin{array}{l}\text { O'Connor et } \\
\text { al., } 2015 \\
\text { UK }\end{array}$ & $\begin{array}{l}\text { The EPIC- } \\
\text { Norfolk study } \\
24,653 \text { men and } \\
\text { women } \\
40-79 \text { years } \\
10.8 \text { years of } \\
\text { follow-up }\end{array}$ & $\begin{array}{l}\text { Sugar-sweetened } \\
\text { soft drinks } \\
\text { Sweetened tea or } \\
\text { coffee } \\
\text { Beverages with } \\
\text { sweetened milk } \\
\text { Artificially sweetened } \\
\text { beverages and } \\
\text { Serving: soft drinks } \\
\text { and artificially } \\
\text { sweetened } \\
\text { beverages, } 336 \\
\text { g/day; tea or coffee } \\
\text { and sugar-sweetened } \\
\text { milk drinks, } 280 \\
\text { g/day; fruit juice, } 150 \\
\text { g/day }\end{array}$ & $\begin{array}{l}1 \\
\text { serving/day }\end{array}$ & Diabetes & $\begin{array}{l}\text { Age, sex, } \\
\text { occupational social } \\
\text { class, education, } \\
\text { family history of } \\
\text { diabetes, physical } \\
\text { activity, smoking, } \\
\text { alcohol consumption, } \\
\text { intake of other sugary } \\
\text { drinks and various } \\
\text { food items, total } \\
\text { energy intake, BMI } \\
\text { and waist } \\
\text { circumference }\end{array}$ & $\begin{array}{l}\text { Soft drinks } \\
(336 \mathrm{~g} / \text { day) } \\
\text { HR } 1.14 \text { (1.01 - } \\
1.32) \\
\text { Beverages with } \\
\text { sweetened milk } \\
(280 \mathrm{~g} / \text { day) } \\
\text { HR } 1.27 \text { (1.09- } \\
1.48) \\
\text { Sweetened tea or } \\
\text { coffee } \\
\text { (280g/day) } \\
\text { HR } 1.03 \text { (0.99- } \\
1.07)\end{array}$ \\
\hline $\begin{array}{l}\text { Micha et } \\
\text { al., } 2017 \\
\text { United } \\
\text { States }\end{array}$ & $\begin{array}{l}\text { National Health } \\
\text { and Nutrition } \\
\text { Examination } \\
\text { Surveys } \\
\text { (NHANES) } \\
16,620 \text { men and } \\
\text { women } \\
\geq 25 \text { years } \\
13 \text { years of } \\
\text { follow-up }\end{array}$ & $\begin{array}{l}\text { Sugar-sweetened } \\
\text { beverages } \\
8 \mathrm{oz} / \text { day }\end{array}$ & $\begin{array}{l}1 \\
\text { serving/day } \\
(80 z)\end{array}$ & $\begin{array}{l}\text { Diabetes } \\
\text { CHD } \\
\text { Stroke }\end{array}$ & $\begin{array}{l}\text { Sex, age, race, } \\
\text { education, energy } \\
\text { intake and BMI }\end{array}$ & $\begin{array}{l}\text { Sugar-sweetened } \\
\text { beverages in } 50- \\
\text { year-olds } \\
\text { (8oz/day) } \\
\text { RR } 1.27 \text { ( } 1.11 \text { - } \\
1.46 \text { ) diabetes } \\
\text { RR } 1.26 \text { (1.15- } \\
1.37 \text { ) CHD } \\
\begin{array}{l}\text { No information on } \\
\text { beverages for } \\
\text { stroke }\end{array}\end{array}$ \\
\hline $\begin{array}{l}\text { Papier et } \\
\text { al., } 2017 \\
\text { Thailand }\end{array}$ & $\begin{array}{l}\text { Thai Cohort } \\
\text { Study (TCS) } \\
39,175 \text { men and } \\
\text { women } \\
\text { Average age } \\
\text { among men ( } 33 \\
\text { y/o) and women } \\
(28 \text { y/o) } \\
8 \text { years of } \\
\text { follow-up }\end{array}$ & $\begin{array}{l}\text { Any sweetened } \\
\text { carbonated beverage } \\
\text { or soft drink }\end{array}$ & $\geq 1$ per day & Diabetes & $\begin{array}{l}\text { Age, area of } \\
\text { residence, education, } \\
\text { income, physical } \\
\text { activity, consumption } \\
\text { of fruits/vegetables, } \\
\text { fried foods and } \\
\text { alcohol, smoking, } \\
\text { hypertension at onset } \\
\text { and baseline BMI }\end{array}$ & $\begin{array}{l}\text { Sugar-sweetened } \\
\text { soft drinks } \\
(\geq 1 \text { per day) } \\
\text { OR } 2.4(1.5-3.9) \\
\text { women } \\
\text { OR } 1.3(0.9-2.1) \\
\text { men }\end{array}$ \\
\hline
\end{tabular}

RR - Relative risk; HR - Hazard ratio; OR - Odds ratio; BMI - Body Mass Index; CHD - Coronary heart disease. 


\begin{tabular}{|c|c|c|c|c|c|c|}
\hline $\begin{array}{l}\text { Author, } \\
\text { year, } \\
\text { country }\end{array}$ & Sample & Drinks included & Cut-off point & Outcome & $\begin{array}{l}\text { Confounders used in } \\
\text { the analysis }\end{array}$ & Main results \\
\hline $\begin{array}{l}\text { Drouin- } \\
\text { Chartier et } \\
\text { al., } 2019 \\
\text { United } \\
\text { States }\end{array}$ & $\begin{array}{l}\text { Nurses' Health } \\
\text { Study (NHS) } \\
76,531 \text { women } \\
30 \text { - } 55 \text { years } \\
26 \text { years of } \\
\text { follow-up } \\
\text { and } \\
\text { NHS II } 81,597 \\
\text { women } \\
25 \text { - } 42 \text { years } \\
22 \text { years of } \\
\text { follow-up } \\
\text { and } \\
\text { Health } \\
\text { Professionals' } \\
\text { Follow-up Study } \\
\text { (HPFS) } \\
34,224 \text { men } \\
40 \text { - } 75 \text { years } \\
26 \text { years of } \\
\text { follow-up }\end{array}$ & $\begin{array}{l}\text { Sugar-sweetened } \\
\text { carbonated or non- } \\
\text { carbonated } \\
\text { beverages (soda, } \\
\text { punch, lemonade, } \\
\text { fruit juice or sugar- } \\
\text { sweetened iced tea) } \\
100 \% \text { fruit juice } \\
\text { Artificially sweetened } \\
\text { beverages } \\
\text { Serving: } 240 \mathrm{ml} / \text { day } \\
8 \mathrm{oz}\end{array}$ & $\begin{array}{l}\text { Change from } \\
1 \\
\text { serving/week } \\
\text { to } 1 \\
\text { serving/day } \\
\text { over } 4 \text { years }\end{array}$ & Diabetes & $\begin{array}{l}\text { Race, family history } \\
\text { of diabetes, physical } \\
\text { examination during a } \\
\text { 4-year cycle, early } \\
\text { menopausal status } \\
\text { and postmenopausal } \\
\text { hormone level (NHS } \\
\text { and NHSII), oral } \\
\text { contraceptive use } \\
\text { (NHSII), simultaneous } \\
\text { change in smoking } \\
\text { status, onset and } \\
\text { change in level of } \\
\text { physical activity, } \\
\text { onset and change in } \\
\text { alcohol consumption, } \\
\text { onset and change in } \\
\text { Alternative Healthy } \\
\text { Eating Index (AHEI) } \\
\text { score, onset and } \\
\text { change in intake of } \\
\text { water, coffee, tea, } \\
\text { milk, sugar- } \\
\text { sweetened and } \\
\text { artificial beverages, } \\
\text { baseline BMI and } \\
\text { baseline calorie } \\
\text { intake }\end{array}$ & $\begin{array}{l}\text { Sugar-sweetened } \\
\text { beverages } \\
(1 / \text { day) } \\
\text { RR } 1.15 \text { (0.98 - } \\
1.35)\end{array}$ \\
\hline $\begin{array}{l}\text { Hirahatake } \\
\text { et al., } 2019 \\
\text { United } \\
\text { States }\end{array}$ & $\begin{array}{l}\text { Coronary Artery } \\
\text { Risk } \\
\text { Development in } \\
\text { Young Adults } \\
\text { (CARDIA) study } \\
4,719 \text { men and } \\
\text { women } \\
18 \text { - } 30 \text { years } \\
30 \text { years of } \\
\text { follow-up }\end{array}$ & $\begin{array}{l}\text { Sugar-sweetened } \\
\text { soft drinks and fruit } \\
\text { drinks } \\
\text { Artificially sweetened } \\
\text { beverages e.g., soda } \\
\text { and fruit juices } \\
\text { sweetened with non- } \\
\text { nutritive sweeteners } \\
\text { Serving: } 8 \text { oz/day }\end{array}$ & $\begin{array}{l}1 \\
\text { serving/day }\end{array}$ & Diabetes & $\begin{array}{l}\text { Education, smoking, } \\
\text { dietary behavior, } \\
\text { mean energy intake, } \\
\text { cumulative physical } \\
\text { activity, mean aMED } \\
\text { score, baseline BMI, } \\
\text { change in weight } \\
\text { from baseline to } \\
\text { diabetes diagnosis, } \\
\text { and end of follow-up }\end{array}$ & $\begin{array}{l}\text { Sugar-sweetened } \\
\text { beverages } \\
\text { (1 serving/day) } \\
\text { HR } 1.06 \text { ( } 1.01- \\
1.10) \\
(\geq 2 / \text { servings/day) } \\
\text { HR } 1.27 \text { (0.93- } \\
1.74)\end{array}$ \\
\hline $\begin{array}{l}\text { Stern et al., } \\
2019 \\
\text { Mexico }\end{array}$ & $\begin{array}{l}\text { Mexican } \\
\text { Teachers' Cohort } \\
\text { (MTC) } \\
76,667 \text { women } \\
\geq 25 \text { years } \\
7 \text { years of } \\
\text { follow-up }\end{array}$ & $\begin{array}{l}\text { Sugar-sweetened } \\
\text { soft drink (cola- } \\
\text { flavored soda or } \\
\text { flavored soda) } \\
\text { Serving: } 355 \mathrm{ml}\end{array}$ & $\begin{array}{l}1 \\
\text { serving/day }\end{array}$ & Diabetes & $\begin{array}{l}\text { Age, region of } \\
\text { residence, inventory } \\
\text { of household goods, } \\
\text { smoking, family } \\
\text { history of diabetes, } \\
\text { physical activity, food } \\
\text { and beverage groups, } \\
\text { and baseline BMI }\end{array}$ & $\begin{array}{l}\text { Sugar-sweetened } \\
\text { soft drink } \\
\text { (1 serving/day) } \\
\text { HR } 1.27 \text { (1.16 - } \\
1.38 \text { ) }\end{array}$ \\
\hline
\end{tabular}

Studies which analyzed SSB intake in grams, milliliter or ounce instead portion also found positive associations with type 2 diabetes. Four different studies showed an increased risk of type 2 diabetes as increased the consumption of SSB, independent of sociodemographic and nutritional characteristics, with effect measures varying from 1.18 RR to 1.34 HR [37], [38], [40], [45]. One of these studies also adjusted the analyses for waist circumference [38], suggesting that the effects of SSB intake on risk of type 2 diabetes appear to be independent of central adiposity. 
The positive effects of SSB intake on the risk of type 2 diabetes was also observed in studies conducted in middle-income countries. Stern et al. [42], analyzing data from almost 80,000 women from the Mexican Teachers' Cohort, found that the consumption of one portion of sweetened soft drinks per day increased the hazard of type 2 diabetes in 1.27 times (HR $=1.27 ; 95 \mathrm{C}$.I. $1.16-1.38)$, after adjusting for several confounders, including dietary intake and body mass index. In 40,000 Chinese Singaporeans, SSB intake increased the risk of type 2 diabetes, independent of lifestyle and dietary confounders [43]. The authors also found that 5-year weight gain was an effect modifier of the association between SSB intake and type 2 diabetes. Finally, data from a cohort study from Thailand [39], observed that SSB intake increased the risk of type 2 diabetes more than two times, independent of demographic and nutritional characteristics. However, positive results were found for women only.

\section{Association between SSB intake and obesity}

The effect of SSB intake on obesity status was investigated by four studies with three of them presenting positive associations [46]-[48] and one with null results [49] (Table 3). In the Framingham Heart Study [46], the consumption of one or more soft drinks per day increased the odds of obesity by $31 \%(\mathrm{OR}=1.31 ; 95 \%$ C.I. $1.02 ; 1.68)$, while the consumption of two or more soft drinks per day increased the odds by $50 \%(\mathrm{OR}=1.50 ; 95 \%$ C.I. 1.06; 2.11). Data from the Korean National Health and Examination Survey [47] showed that men and women who have consumed one or more portion of SSB per day presented higher odds of obesity when compared to those who consumed less than one portion per day. Both studies adjusted analyses for several confounders, but only the first one adjusted for dietary characteristics. 
Table 3

Description of studies selected by systematic review about the longitudinal effects of sugar sweetened beverages intake on obesity, coronary heart disease and stroke in adults.

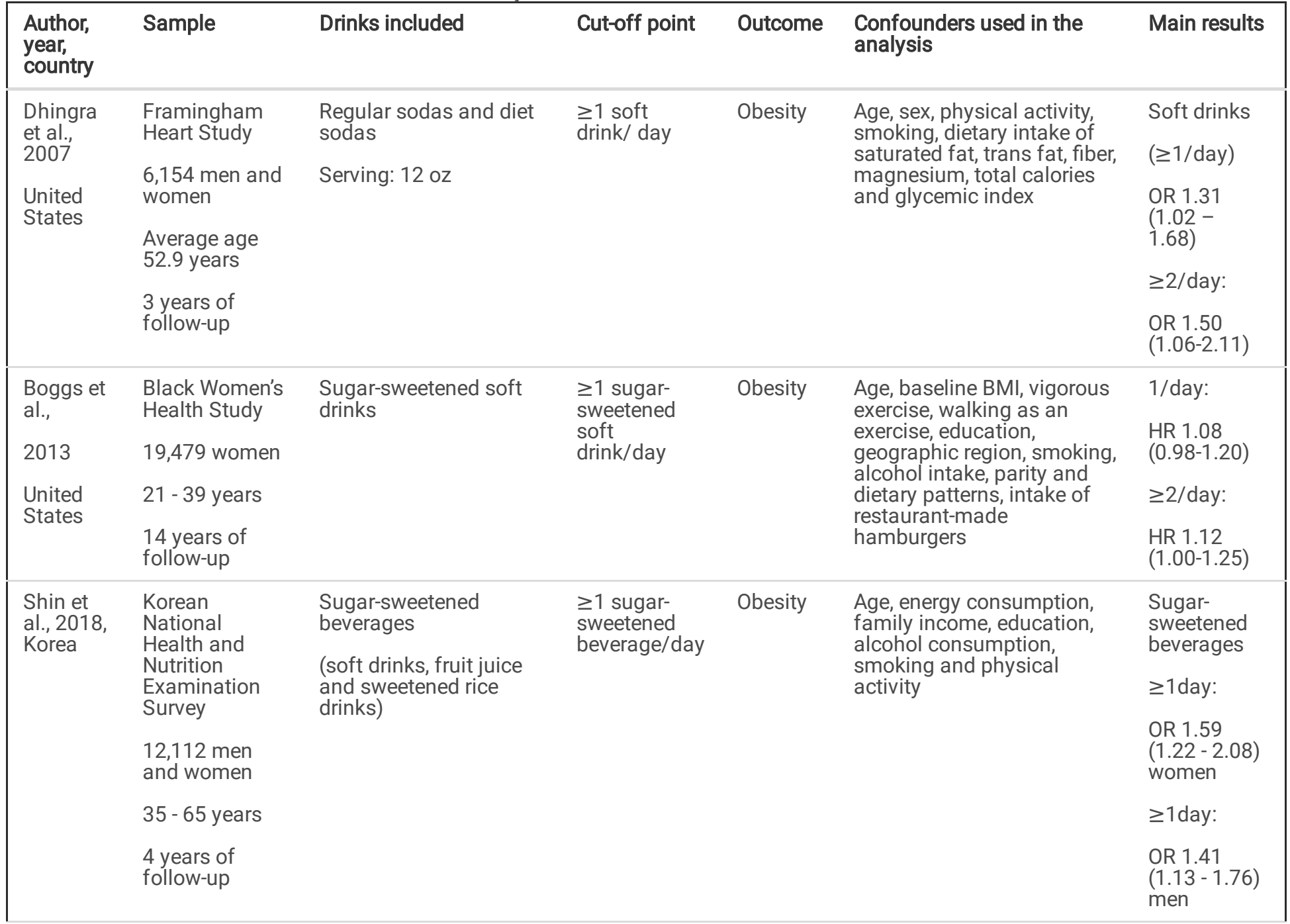




\begin{tabular}{|c|c|c|c|c|c|c|}
\hline $\begin{array}{l}\text { Author, } \\
\text { year, } \\
\text { country }\end{array}$ & Sample & Drinks included & Cut-off point & Outcome & $\begin{array}{l}\text { Confounders used in the } \\
\text { analysis }\end{array}$ & Main results \\
\hline \multirow{13}{*}{$\begin{array}{l}\text { Garduno- } \\
\text { Alanis et } \\
\text { al., } 2020 \\
\text { Eastern } \\
\text { Europe }\end{array}$} & $\begin{array}{l}\text { The HAPIEE } \\
\text { Study }\end{array}$ & $\begin{array}{l}\text { Sugar-sweetened } \\
\text { beverages (soft drinks) }\end{array}$ & \multirow{13}{*}{$\begin{array}{l}\geq 1 \text { sugar- } \\
\text { sweetened } \\
\text { beverage/day }\end{array}$} & \multirow[t]{13}{*}{ Obesity } & \multirow{13}{*}{$\begin{array}{l}\text { Age, sex, education, marital } \\
\text { status, smoking, alcohol } \\
\text { consumption, physical } \\
\text { activity, energy intake, fruit } \\
\text { and vegetable consumption } \\
\text { and previous diagnoses of } \\
\text { chronic diseases such as } \\
\text { diabetes, CVD or cancer }\end{array}$} & \multirow{2}{*}{$\begin{array}{l}\text { Sugar- } \\
\text { sweetened } \\
\text { beverages } \\
(\geq 1 / \text { day })\end{array}$} \\
\hline & $\begin{array}{l}26,634 \text { men } \\
\text { and women }\end{array}$ & \multirow[t]{2}{*}{$\begin{array}{l}\text { Artificially sweetened } \\
\text { soft drinks }\end{array}$} & & & & \\
\hline & $45-69$ years & & & & & $\begin{array}{l}\text { Total sugar- } \\
\text { sweetened }\end{array}$ \\
\hline & \multirow[t]{10}{*}{$\begin{array}{l}3 \text { years of } \\
\text { follow-up }\end{array}$} & \multirow[t]{10}{*}{ Serving: $200 \mathrm{ml}$} & & & & ( $\geq 1 /$ day) \\
\hline & & & & & & $\begin{array}{l}\text { Czech } \\
\text { Republic }\end{array}$ \\
\hline & & & & & & OR 0.28 \\
\hline & & & & & & $(0.02-0.54)$ \\
\hline & & & & & & Russia \\
\hline & & & & & & OR 1.38 \\
\hline & & & & & & $(0.62-2.15)$ \\
\hline & & & & & & Poland \\
\hline & & & & & & OR 0.83 \\
\hline & & & & & & $(0.29-1.37)$ \\
\hline \multirow{9}{*}{$\begin{array}{l}\text { Fung et } \\
\text { al., } 2009 \\
\text { United } \\
\text { States }\end{array}$} & $\begin{array}{l}\text { Nurses 'Health } \\
\text { Study (NHS) }\end{array}$ & \multirow{3}{*}{$\begin{array}{l}\text { Beverages sweetened } \\
\text { with cola sugar, } \\
\text { caffeinated and } \\
\text { caffeine-free } \\
\text { Other carbonated sugar- } \\
\text { sweetened beverages }\end{array}$} & \multirow[t]{9}{*}{$\begin{array}{l}\geq 2 \\
\text { servings/day }\end{array}$} & \multirow[t]{9}{*}{$\begin{array}{l}\text { Coronary } \\
\text { disease }\end{array}$} & \multirow{9}{*}{$\begin{array}{l}\text { Age, smoking, alcohol } \\
\text { intake, parental history of } \\
\text { myocardial infarction } \\
\text { before } 60 \text { years of age, } \\
\text { physical activity, aspirin } \\
\text { use, menopausal status } \\
\text { and postmenopausal } \\
\text { hormone use, history of } \\
\text { hypertension and high } \\
\text { cholesterol and alternative } \\
\text { healthy eating index (AHEI) }\end{array}$} & $\begin{array}{l}\text { Total } \\
\text { sweetened }\end{array}$ \\
\hline & \multirow{8}{*}{$\begin{array}{l}34 \text { - } 59 \text { years } \\
24 \text { years of } \\
\text { follow-up }\end{array}$} & & & & & \\
\hline & & & & & & 2/day) \\
\hline & & \multirow{6}{*}{$\begin{array}{l}\text { Non-carbonated } \\
\text { sweetened beverages } \\
\text { Artificially sweetened } \\
\text { beverages }\end{array}$} & & & & $\begin{array}{l}\text { RR 1.23 } \\
(1.06-1.43)\end{array}$ \\
\hline & & & & & & ( $\geq 2 /$ day) \\
\hline & & & & & & $\begin{array}{l}\text { RR } 1.35 \\
(1.07-1.69)\end{array}$ \\
\hline & & & & & & $\begin{array}{l}\text { Total sugar- } \\
\text { sweetened } \\
\text { beverages }\end{array}$ \\
\hline & & & & & & (2/day) \\
\hline & & & & & & $\begin{array}{l}\text { RR } 1.28 \\
(1.14-1.44)\end{array}$ \\
\hline \multirow{5}{*}{$\begin{array}{l}\text { de } \\
\text { Koning } \\
\text { et al., } \\
2012 \\
\text { United } \\
\text { States }\end{array}$} & $\begin{array}{l}\text { Health } \\
\text { Professionals } \\
\text { Follow-Up } \\
\text { Study (HPFS) }\end{array}$ & $\begin{array}{l}\text { Sugar-sweetened cola } \\
\text { drinks (caffeinated and } \\
\text { caffeine-free) }\end{array}$ & \multirow[t]{5}{*}{1 serving/day } & \multirow[t]{5}{*}{$\begin{array}{l}\text { Coronary } \\
\text { disease }\end{array}$} & \multirow{5}{*}{$\begin{array}{l}\text { Smoking, physical activity, } \\
\text { alcohol intake, multivitamin } \\
\text { use, family history of } \mathrm{CHD} \text {, } \\
\text { pre-enrollment weight gain, } \\
\text { weight loss, adherence to a } \\
\text { low-calorie diet, }\end{array}$} & $\begin{array}{l}\text { Total sugar- } \\
\text { sweetened } \\
\text { beverages }\end{array}$ \\
\hline & $42,883 \mathrm{men}$ & $\begin{array}{l}\text { Other sugar-sweetened } \\
\text { carbonated beverages }\end{array}$ & & & & (1/day) \\
\hline & \multirow[t]{3}{*}{$40-75$ years } & $\begin{array}{l}\text { Sugar-sweetened non- } \\
\text { carbonated beverages } \\
\text { (fruit punch, lemonade } \\
\text { or fruit drinks) }\end{array}$ & & & & \multirow[t]{3}{*}{$\begin{array}{l}\text { RR } 1.19 \\
(1.11-1.28)\end{array}$} \\
\hline & & $\begin{array}{l}\text { Artificially sweetened } \\
\text { beverages }\end{array}$ & & & & \\
\hline & & Serving: 355 ml (12 oz) & & & & \\
\hline
\end{tabular}




\begin{tabular}{|c|c|c|c|c|c|c|}
\hline $\begin{array}{l}\text { Author, } \\
\text { year, } \\
\text { country }\end{array}$ & Sample & Drinks included & Cut-off point & Outcome & $\begin{array}{l}\text { Confounders used in the } \\
\text { analysis }\end{array}$ & Main results \\
\hline $\begin{array}{l}\text { Keller et } \\
\text { al., } 2020 \\
\text { United } \\
\text { States }\end{array}$ & $\begin{array}{l}\text { Harvard } \\
\text { Pooling Project } \\
\text { (HPP) } \\
284,345 \text { men } \\
\text { and women } \\
\geq 35 \text { years }\end{array}$ & $\begin{array}{l}\text { Sugar-sweetened } \\
\text { beverages } \\
\text { (carbonated/non- } \\
\text { carbonated and } \\
\text { caffeinated/caffeine- } \\
\text { free soft drinks, sports } \\
\text { drinks and fruit drinks } \\
\text { with any type of added } \\
\text { sugar) } \\
\text { All types of coffee } \\
\text { All types of tea } \\
\text { (unsweetened) } \\
\text { Unsweetened, whole or } \\
\text { low-fat cow's milk } \\
\text { 100\% fruit juice } \\
\text { Artificially sweetened } \\
\text { beverages } \\
\text { Serving: } 355 \text { ml (12 oz) }\end{array}$ & 1 serving/day & $\begin{array}{l}\text { Coronary } \\
\text { disease }\end{array}$ & $\begin{array}{l}\text { Nutrient intake, total energy } \\
\text { intake, BMl, history of } \\
\text { hypertension and high } \\
\text { cholesterol, smoking, } \\
\text { physical activity, education } \\
\text { and alcohol consumption }\end{array}$ & 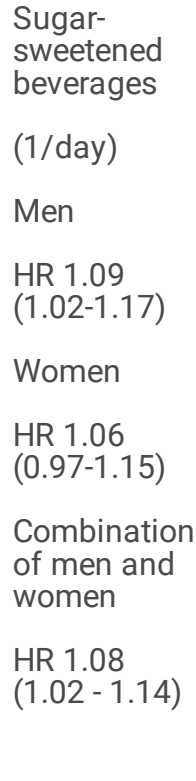 \\
\hline $\begin{array}{l}\text { Bernstein } \\
\text { et al., } \\
2012 \\
\text { United } \\
\text { States }\end{array}$ & $\begin{array}{l}\text { Nurses 'Health } \\
\text { Study (NHS) } \\
84,085 \text { women } \\
\text { aged } 30 \text { to } 55 \\
\text { years old } \\
28 \text { years of } \\
\text { follow-up } \\
\text { and } \\
\text { Health } \\
\text { Professionals } \\
\text { Follow-Up } \\
\text { Study (HPFS) } \\
43,371 \text { men } \\
40-75 \text { years } \\
22 \text { years of } \\
\text { follow-up }\end{array}$ & $\begin{array}{l}\text { Caffeine-free and low- } \\
\text { calorie cola } \\
\text { Other low-calorie } \\
\text { carbonated beverages } \\
\text { Sugar-sweetened, } \\
\text { caffeinated and } \\
\text { caffeine-free cola } \\
\text { Other sugar-sweetened } \\
\text { carbonated beverages }\end{array}$ & $\begin{array}{l}\geq 1 \\
\text { serving/day }\end{array}$ & Stroke & $\begin{array}{l}\text { Other types of beverages, } \\
\text { age, calendar time, various } \\
\text { food items and nutrients, } \\
\text { physical activity, smoking, } \\
\text { menopausal status in } \\
\text { women, parental history of } \\
\text { early myocardial infarction, } \\
\text { multivitamin use, vitamin E } \\
\text { supplement use, and aspirin } \\
\text { use at least once a week. } \\
\text { BMl, energy intake and } \\
\text { weight change }\end{array}$ & $\begin{array}{l}\begin{array}{l}\text { Sugar- } \\
\text { sweetened } \\
\text { beverages }\end{array} \\
(1 / \text { day }) \\
\text { Men } \\
\text { RR } 1.08 \\
(0.89-1.32) \\
\text { Women } \\
\text { RR } 1.14 \\
(1.02-1.27)\end{array}$ \\
\hline $\begin{array}{l}\text { Eshak et } \\
\text { al., } 2012 \\
\text { Japan }\end{array}$ & $\begin{array}{l}43,149 \text { men } \\
\text { and women } \\
\text { aged } 40 \text { to } 59 \\
\text { years old } \\
18 \text { years of } \\
\text { follow-up }\end{array}$ & $\begin{array}{l}\text { Soft drinks (cola, } \\
\text { flavored juices and non- } \\
100 \% \text { fruit juices) } \\
\text { Serving: } 250 \mathrm{~g}\end{array}$ & $\begin{array}{l}\text { Nearly daily } \\
\text { intake } \\
(\geq 1 \\
\text { glass/day) }\end{array}$ & Stroke & $\begin{array}{l}\text { Age, history of } \\
\text { hypertension, history of } \\
\text { diabetes, smoking, alcohol } \\
\text { intake, leisure-time physical } \\
\text { activity, work status, dietary } \\
\text { intake quintiles adjusted for } \\
\text { energy from selected foods } \\
\text { and nutrients, BMI, and total } \\
\text { energy intake }\end{array}$ & $\begin{array}{l}\text { Men } \\
\text { Stroke } \\
\text { HR } 0.76 \\
(0.62,1.06) \\
\text { Women } \\
\text { Stroke } \\
\text { HR } 1.21 \\
(0.88,1.68)\end{array}$ \\
\hline
\end{tabular}




\begin{tabular}{|c|c|c|c|c|c|c|}
\hline $\begin{array}{l}\text { Author, } \\
\text { year, } \\
\text { country }\end{array}$ & Sample & Drinks included & Cut-off point & Outcome & $\begin{array}{l}\text { Confounders used in the } \\
\text { analysis }\end{array}$ & Main results \\
\hline \multirow[t]{18}{*}{$\begin{array}{l}\text { Larsson } \\
\text { et al., } \\
2014\end{array}$} & \multirow{5}{*}{$\begin{array}{l}\text { The Swedish } \\
\text { Mammography } \\
\text { Cohort } \\
32,575 \text { women } \\
49 \text { - } 83 \text { years }\end{array}$} & \multirow{18}{*}{$\begin{array}{l}\text { Sugary drinks (soft } \\
\text { drinks and juice) } \\
\text { Coffee, tea, milk } \\
\text { Serving: } 200 \mathrm{ml}\end{array}$} & \multirow[t]{18}{*}{$\begin{array}{l}\geq 1 \\
\text { serving/day }\end{array}$} & \multirow[t]{18}{*}{ Stroke } & \multirow{18}{*}{$\begin{array}{l}\text { Education, family history of } \\
\text { early myocardial infarction, } \\
\text { smoking, physical activity, } \\
\text { history of hypertension, } \\
\text { aspirin use, total energy } \\
\text { intake, consumption of } \\
\text { alcohol and various } \\
\text { beverages and food items, } \\
\text { and waist circumference }\end{array}$} & $\begin{array}{l}\text { Sugar- } \\
\text { sweetened }\end{array}$ \\
\hline & & & & & & (1 to $<$ \\
\hline & & & & & & 2/day) \\
\hline & & & & & & Women \\
\hline & & & & & & $\begin{array}{l}1.06(0.88- \\
1.30)\end{array}$ \\
\hline & & & & & & Men \\
\hline & \multirow{2}{*}{$\begin{array}{l}\text { Cohort of } \\
\text { Swedish Men }\end{array}$} & & & & & $\begin{array}{l}1.17(0.98- \\
1.39)\end{array}$ \\
\hline & & & & & & \multirow{3}{*}{$\begin{array}{l}\text { Combination } \\
\text { between } \\
\text { men and } \\
\text { women }\end{array}$} \\
\hline & 35,884 men & & & & & \\
\hline & $45-79$ years & & & & & \\
\hline & \multirow[t]{8}{*}{$\begin{array}{l}10.3 \text { years of } \\
\text { follow-up }\end{array}$} & & & & & $\begin{array}{l}\text { RR } 1.12 \\
(0.99-1.28)\end{array}$ \\
\hline & & & & & & ( $\geq 2 /$ day) \\
\hline & & & & & & Women \\
\hline & & & & & & $\begin{array}{l}1.14(0.92- \\
1.41)\end{array}$ \\
\hline & & & & & & Men \\
\hline & & & & & & $\begin{array}{l}1.22(1.02- \\
1.45)\end{array}$ \\
\hline & & & & & & $\begin{array}{l}\text { Combination } \\
\text { between } \\
\text { men and } \\
\text { women }\end{array}$ \\
\hline & & & & & & $\begin{array}{l}\text { RR } 1.19 \\
(1.04-1.36)\end{array}$ \\
\hline \multirow{7}{*}{$\begin{array}{l}\text { Pase et } \\
\text { al., } 2017 \\
\text { United } \\
\text { States }\end{array}$} & \multirow{3}{*}{$\begin{array}{l}\text { Framingham } \\
\text { Heart Study } \\
\text { Offspring } \\
\text { 2,888 men and } \\
\text { women }\end{array}$} & \multirow{2}{*}{$\begin{array}{l}\text { Sugary beverages } \\
\text { (sugar-sweetened soft } \\
\text { drinks, fruit juice and } \\
\text { fruit drinks) }\end{array}$} & \multirow[t]{7}{*}{$\begin{array}{l}1-2 \\
\text { servings/day }\end{array}$} & \multirow[t]{7}{*}{ Stroke } & \multirow{7}{*}{$\begin{array}{l}\text { Age, gender, education, total } \\
\text { caloric intake, dietary } \\
\text { guideline adherence index } \\
\text { (DGAl), physical activity, } \\
\text { smoking, and } \\
\text { cardiometabolic variables }\end{array}$} & $\begin{array}{l}\text { Total sugar- } \\
\text { sweetened } \\
\text { beverages }\end{array}$ \\
\hline & & & & & & (1-2/day) \\
\hline & & \multirow{2}{*}{$\begin{array}{l}\text { Sugar-sweetened soft } \\
\text { drinks (carbonated, with } \\
\text { high sugar content, e.g., } \\
\text { cola) }\end{array}$} & & & & HR 1.22 \\
\hline & $\geq 45$ years & & & & & $(0.77-1.94)$ \\
\hline & \multirow{3}{*}{$\begin{array}{l}10 \text { years of } \\
\text { follow-up }\end{array}$} & Artificially sweetened & & & & (> 2/day) \\
\hline & & soft drinks & & & & \multirow{2}{*}{$\begin{array}{l}\text { HR } 0.88 \\
(0.42-1.83)\end{array}$} \\
\hline & & Fruit juice & & & & \\
\hline
\end{tabular}




\begin{tabular}{|c|c|c|c|c|c|c|}
\hline $\begin{array}{l}\text { Author, } \\
\text { year, } \\
\text { country }\end{array}$ & Sample & Drinks included & Cut-off point & Outcome & $\begin{array}{l}\text { Confounders used in the } \\
\text { analysis }\end{array}$ & Main results \\
\hline \multirow{4}{*}{$\begin{array}{l}\text { Pacheco } \\
\text { et al., } \\
2020 \\
\text { United } \\
\text { States }\end{array}$} & $\begin{array}{l}\text { California } \\
\text { Teachers Study }\end{array}$ & $\begin{array}{l}\text { Regular sodas and fruit- } \\
\text { based sodas }\end{array}$ & \multirow[t]{4}{*}{$\begin{array}{l}\geq 1 \\
\text { serving/day }\end{array}$} & \multirow[t]{4}{*}{ Stroke } & \multirow{4}{*}{$\begin{array}{l}\text { Age, race, socioeconomic } \\
\text { status, smoking, alcohol } \\
\text { intake, family history of } \\
\text { cardiovascular disease, } \\
\text { physical activity, aspirin } \\
\text { use, multivitamin use, } \\
\text { menopausal status and } \\
\text { hormone therapy use, oral } \\
\text { contraceptive use, history of } \\
\text { hypertension, BMl, total } \\
\text { energy intake and fruit and } \\
\text { vegetable intake }\end{array}$} & \multirow{4}{*}{ 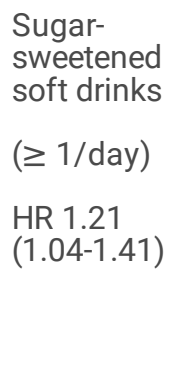 } \\
\hline & $\begin{array}{l}106,178 \\
\text { women }\end{array}$ & \multirow{2}{*}{$\begin{array}{l}\text { Serving: } 355 g(12 \mathrm{oz}) \\
\text { Sweetened bottled } \\
\text { water or iced tea, other } \\
\text { beverages with added } \\
\text { vitamin C }\end{array}$} & & & & \\
\hline & $\begin{array}{l}\text { Average age } \\
51.2\end{array}$ & & & & & \\
\hline & $\begin{array}{l}20 \text { years of } \\
\text { follow-up }\end{array}$ & Serving: $237 \mathrm{~g}(8 \mathrm{oz})$ & & & & \\
\hline
\end{tabular}

RR - Relative risk; HR - Hazard ratio; OR - Odds ratio; BMI - Body Mass Index; CHD - Coronary heart disease; CVD - Cardiovascular disease.

Garduno-Alanis et al. [48], analyzing data from the Health, Alcohol and Psychosocial factors in Eastern Europe, found that SSB intake increased the risk of obesity, independent of several confounders, including dietary characteristics as well as medical history of CVD, cancer and diabetes. Interesting to notice that results were only significant in Poland and Russia, but not in Czech Republic.

\section{Association between SSB intake and coronary heart disease}

Three longitudinal studies included in our systematic review hypothesized that SSB intake is a risk factor for CHD (Table 3). One study included only women in the sample [50], one study enrolled only men [51], and the other one included both men and women in the sample [52]. The investigation that analyzed data for men and women from the Harvard Pooling Project observed that the intake of more than one portion of SSB per day increased the hazard of CHD (HR $=1.08 ; 95 \%$ C.I. 1.02; 1.14). Stratification by sex revealed that results were significant for men but not for women [52].

In addition, the consumption of two or more portions of SSB per day increased almost $30 \%$ the risk of CHD in 90 thousand women from the Nurses' Health Study [50]. The authors adjusted the analyses for several confounders, including lifestyle and nutritional characteristics, and health status. De Koning et al. [51], in turn, found that more than one portion of SSB increased by almost $20 \%$ the risk of $\mathrm{CHD}(\mathrm{RR}=1.19 ; 95 \%$ C.I. $1.11-1.28)$ in more than 40 thousand men from the Health Professionals Follow-up Study. The results were also adjusted for several confounders.

\section{Association between SSB intake and stroke}

Five studies investigated the association between SSB intake and stroke [53]- [57] (Table 3). Larsson et al. [54], analyzing data from the Cohort of Swedish, showed that the consumption of two or more portions of SSB was associated with higher risk of stroke $(R R=1.19$; $95 \%$ C.I. $1.04 ; 1.36)$. Nevertheless, when they stratified results by sex, association was significant for men only $(R R=1.22 ; 95 \%$ C.I. 1.02 ; 1.36). The authors adjusted the results for several confounders including dietary characteristics and waist circumference, suggesting that the effect of SSB on stroke may be independent of central adiposity.

Bernstein et al. [57] and Pacheco et al. [56], on the other hand, found significant results for women only. The first study observed that the consumption of one or more portion of SSB increased the risk of stroke only in women (RR $=1.14 ; 95 \%$ C.I. 1.02; 1.27), after 28 years of follow-up [57]. Similarly, one or more portion of SSB per day (more than $355 \mathrm{~g}$ ) increased the hazard of stroke in more than 100,000 women, after adjustment for several confounders, including dietary characteristics and body mass index [56].

Nevertheless, two longitudinal studies did not observed effects of SSB intake on the risk of stroke after controlling for potential confounders in 40,000 men and women from Japan [53] or in almost 3000 men and women from US [55], after 18 and 10 years of follow-up, respectively.

\section{Meta analysis}

From the 27 studies selected in the systematic review, 26 were included in our meta-analysis as one investigation did not present OR, HR or RR in results. Figure 2 shows that the grouped effect of SSB on obesity and type 2 diabetes was positive. When obesity was the main outcome analyzed, we observed a positive pooled effect of SSB intake on the risk of obesity in the four studies included in the metaanalysis, with low heterogeneity among them $\left(I^{2}=36 \%\right.$; $p$-value $\left.=0.20\right)$. Results comparing low/moderate intake vs. no intake, and high 
intake vs. no intake (available for only two studies) showed that intake of SSB increased the risk of obesity by, on average, $17 \%$ (RR = 1.17; 95\%C.I. $1.10-1.25)$.

We also observed a positive pooled effect of low/moderate intake of SSB on the risk of type 2 diabetes in adults (RR = 1.20; $95 \%$ C.I. 1.13 - 1.28). From the 14 studies included, eight presented positive results. The $\mathrm{I}^{2}$ statistics indicated moderate heterogeneity among studies

$\left(I^{2}=70 \%\right.$; p-value $\left.<0.01\right)$. Results comparing high intake vs. no intake (available for 6 out of 14 studies) showed that the pooled effect was even larger: high intake of SSB increased the risk of diabetes by $32 \%(\mathrm{RR}=1.32 ; 95 \%$ C.I. 1.22; 1.44).

In Figure 3, we have the meta-analysis results for the association between SSB intake and CHD as well as stroke. The pooled effect revealed that SSB intake increased the risk of CHD by $15 \%(R R=1.15 ; 95 \% C . I .1 .06-1.25)$, with moderate heterogeneity among the included studies. When stroke was the outcome, the pooled estimates from the five included studies showed that low/moderate intake of SSB increased the risk of stroke by $10 \%(R R=1.10 ; 95 \%$ C.I. $1.01=1.19)$, with low heterogeneity among studies $\left(I^{2}=43 \%\right.$; $p$-value $\left.=0.14\right)$. Results comparing high intake vs. no intake (available for only three out of five studies) were not statistically significant.

\section{Risk of bias across studies}

Funnel plot (Supplementary figure 1) along with Beeg's and Egger's test showed no evidence of publication bias for the meta-analysis of the effect of SSB intake on diabetes ( $p$-value for Begg \& Mamzudar test $=0.87$; p-value for Egger's test $=0.046$ ). For the other outcomes analyzed in this study (obesity, CHD and stroke), we were not able to test the risk of bias due to the small number of publications included in the meta-analysis [58].

\section{Discussion}

Our systematic review and meta-analysis, which included longitudinal studies with adults, showed considerably consistent results on the association between SSB intake and NCDs. According to the prospective studies included in our review, artificially sugar-sweetened beverages increased the risk of type 2 diabetes, obesity, CHD and stroke, with increased risks of low/moderate SSB intake ranging from $10 \%$ for stroke to $20 \%$ for type 2 diabetes.

Type 2 diabetes was the outcome presenting the strongest association with SSB intake according to our systematic review and metaanalysis. In fact, this is not the first systematic review showing prospective association between SSB intake and type 2 diabetes. Imamura et al. [11] have found that higher consumption of SSB increased the incidence of type 2 diabetes by $18 \%$, with adiposity attenuating this effect $(R R=1.13 ; 95 \%$ C.I. 1.06 to 1.21). The authors also estimated that, over 10 years, two million people developed type 2 diabetes in US and almost 100 thousand in UK due to consumption of SSB [11]. Greenwood et al. [59] have found similar results: SSB intake increased the risk of type 2 diabetes by $20 \%$ (RR 1.20; 95\%C.I. 1.12; 1.29), with the risk being attenuated after adjustment for $\mathrm{BMI}$, indicating that adiposity is involved in the causal pathway. In our review, most studies which included type 2 diabetes as the outcome have adjusted results for BMI or waist circumference, and there were not differences among effect measures of studies adjusting or not adjusting analyses by measures of adiposity.

Results for the other outcomes (obesity, CHD and stroke) were less evident in our review, both due to the smaller number of selected studies as well as due to the smaller magnitude of the associations. In consonance with our findings, previous published reviews have already demonstrated that SSB intake is positively associated with weight gain and cardiovascular diseases [12], [13], [60]. Malik et al. [60] have found that effects of SSB intake on weight gain is observed not solely in adults, but even in children. The authors showed that an increase of one-serving in SSB intake per day incremented weight gain in $0.12 \mathrm{Kg}$ in adults and BMI in $0.05 \mathrm{Kg} / \mathrm{m}^{2}$ in children [60]. In addition, two systematic reviews and meta-analyses of prospective studies have suggested that SSB consumption increases the risk of stroke by $13 \%(R R=1.13 ; 95 \%$ C.I. 1.02 to 1.24$)$ [61] and the risk of $\mathrm{CHD}$ by $17 \%(\mathrm{RR}=1.17 ; 95 \% \mathrm{C} . \mathrm{I}$. 1.07 to 1.28$)$ [62], results quite similar to the ones presented in our study.

SSB intake may increase the risk of NCDs by different causal pathways. The first one is through weight gain and excess adiposity caused by excessive daily energy intake [13]. But weight gain and adiposity are not the only causal pathway involved in this relationship, as most studies included in our review have adjusted the results for anthropometric measures, like BMI and waist circumference, and, even so, results remained significant. SSB intake also increases the risk of NCD via high glycemic load, which, in turn, leads to insulin resistance, inflammation and dyslipidemia [13], [63]-[65]. This pathway may help to better understand why the type 2diabetes was the outcome presenting the strongest association with SSB intake. 
Another interesting result evidenced by our study is the lack of investigations on the longitudinal associations between SSB intake and NCD from LMIC. None of the selected studies were conducted in low-income countries and only three in middle-income countries, which is troublesome since LMIC are the settings with the highest burden of deaths attributable to SSB consumption [14]. Estimates for global consumption of SSB with data from 2010 indicated that Latin America and Caribbean, a region comprised by LMIC, presented the highest rates of SSB intake [8]. Moreover, despite there is current evidence of decreasing in SSB intake, trends information on SSB consumption are mostly available for high-income countries with few published data from LMIC.

There is an increasing concern on the standardization of food classification to assess dietary intake in epidemiological studies [66], [67]. Despite the concerning about that, standardized and harmonized protocols to assess SSB intake have not been developed so far. It becomes more evident when we look on the way SSB intake was assessed and categorized in the included studies. While some investigations evaluated only the frequency of consumption, not considering the portion size, other classified consumption in grams, milliliters or ounce.

But even those studies taking portion size into account, the cut-off to define high consumption also varied. In addition, the reviewed studies included different types of SSB, since solely soft drinks up to beverages with sweetened milk, tea, or coffee. It is important to define a standard approach to assess SSB intake in epidemiological studies to make comparisons easier as well as to objectively define a cut-off of SSB intake that indicates health risks at both individual and populational levels.

Finally, we should also consider that more than a half out of the 27 investigations included in our review followed-up the enrolled individuals for less than 10 years. Despite scarce evidence on how long takes for environmental factors, such as dietary intake, induce NCDs [68], [69], studies following individuals for short periods may not have enough time to address the cumulative exposure of SSB intake associated with the onset or the clinical detection of the diseases analyzed. Considering the long latency period of NCD [70], longitudinal studies should have to consider following the sample for longer periods, which is a challenge in the research practice.

Our study has limitations that are important to be mentioned. The first limitation is the possibility of residual confounding, which is common in observational studies [71]. Even though the included studies have adjusted for several confounders like socioeconomic, dietary and body composition characteristics, errors in measuring these characteristics can exist resulting in residual confounding in our meta-analysis. In addition, gray literature, such as academic thesis and conference papers, is more likely to present null results and have not been included here, which can increase the risk of publication bias [72].

As strengths of our systematic review, we can indicate the inclusion of only longitudinal studies, which decreases the risk of reverse causality in the associations investigated. In addition, our systematic review focused in more than one NCD and find similar results for type 2 diabetes, obesity, CHD and stroke, despite differences in the number of included studies for each outcome. This aspect of our study helps to increase the consistency in the association between SSB intake and NCDs.

In conclusion, longitudinal studies with adults demonstrated that consumption of SSB intake appears to increase the risk of type 2 diabetes, obesity, CHD and stroke. The evidence was stronger for type 2 diabetes, and more studies including obesity, CHD and stroke as the main outcome are needed. In addition, longitudinal studies about the association between SSB intake and NCD in LMIC are crucial to better understand this association in these settings. Political programs and actions are important to be developed to reduce the SSB intake and, by consequence, decrease the worldwide incidence of NCD.

\section{Declarations}

\section{Funding information}

This study was supported by Brazilian National Research Council (CNPq) (grant number 442801/2019-0).

\section{Conflict of interest}

The authors declare they do not have any conflict of interest.

\section{Availability of data and material}

Not applicable.

Author's contribution

Page 19/26 
LPS: Data analysis, drafting, critical revision and editing of the manuscript. RMB: Proposed the idea, supervision of the project and critical revision of the manuscript. FMD and APM: literature search, data analysis and critical revision of the manuscript. DPG: supervision of the project, literature search and critical revision of the manuscript.

\section{Consent for publication}

All authors have read and approved the final version of the manuscript.

\section{References}

[1] H. Dai, T. A. Alsalhe, N. Chalghaf, M. Riccò, N. L. Bragazzi, and J. Wu, "The global burden of disease attributable to high body mass index in 195 countries and territories, 1990-2017: An analysis of the Global Burden of Disease Study," PLOS Med., vol. 17, no. 7, p. e1003198, Jul. 2020, doi: 10.1371/journal.pmed.1003198.

[2] M. A. B. Khan, M. J. Hashim, J. K. King, R. D. Govender, H. Mustafa, and J. Al Kaabi, “Epidemiology of Type 2 Diabetes - Global Burden of Disease and Forecasted Trends:," J. Epidemiol. Glob. Health, vol. 10, no. 1, p. 107, 2019, doi: 10.2991/jegh.k.191028.001.

[3] G. A. Roth et al., "Global Burden of Cardiovascular Diseases and Risk Factors, 1990-2019," J. Am. Coll. Cardiol., vol. 76, no. 25, pp. 2982-3021, Dec. 2020, doi: 10.1016/j.jacc.2020.11.010.

[4] S. M. S. Islam, T. D. Purnat, N. T. A. Phuong, U. Mwingira, K. Schacht, and G. Fröschl, "Non-Communicable Diseases (NCDs) in developing countries: a symposium report," Glob. Health, vol. 10, no. 1, p. 81, Dec. 2014, doi: 10.1186/s12992-014-0081-9.

[5] R. D. Semba, M. W. Bloem, and P. Piot, Eds., Nutrition and Health in Developing Countries. Totowa, NJ: Humana Press, 2008. doi: 10.1007/978-1-59745-464-3.

[6] R. Ronto, J. H. Wu, and G. M. Singh, "The global nutrition transition: trends, disease burdens and policy interventions," Public Health Nutr., vol. 21, no. 12, pp. 2267-2270, Aug. 2018, doi: 10.1017/S1368980018000423.

[7] C. A. Monteiro, J.-C. Moubarac, G. Cannon, S. W. Ng, and B. Popkin, "Ultra-processed products are becoming dominant in the global food system: Ultra-processed products: global dominance,” Obes. Rev., vol. 14, pp. 21-28, Nov. 2013, doi: 10.1111/obr.12107.

[8] G. M. Singh et al., "Global, Regional, and National Consumption of Sugar-Sweetened Beverages, Fruit Juices, and Milk: A Systematic Assessment of Beverage Intake in 187 Countries," PLOS ONE, vol. 10, no. 8, p. e0124845, Aug. 2015, doi: 10.1371/journal.pone.0124845.

[9] The InterAct consortium, "Consumption of sweet beverages and type 2 diabetes incidence in European adults: results from EPICInterAct," Diabetologia, vol. 56, no. 7, pp. 1520-1530, Jul. 2013, doi: 10.1007/s00125-013-2899-8.

[10] F. B. Hu and V. S. Malik, "Sugar-sweetened beverages and risk of obesity and type 2 diabetes: Epidemiologic evidence," Physiol. Behav., vol. 100, no. 1, pp. 47-54, Apr. 2010, doi: 10.1016/j.physbeh.2010.01.036.

[11] F. Imamura et al., "Consumption of sugar sweetened beverages, artificially sweetened beverages, and fruit juice and incidence of type 2 diabetes: systematic review, meta-analysis, and estimation of population attributable fraction," BMJ, p. h3576, Jul. 2015, doi: 10.1136/bmj.h3576.

[12] V. S. Malik, B. M. Popkin, G. A. Bray, J.-P. Després, and F. B. Hu, "Sugar-Sweetened Beverages, Obesity, Type 2 Diabetes Mellitus, and Cardiovascular Disease Risk," Circulation, vol. 121, no. 11, pp. 1356-1364, Mar. 2010, doi: 10.1161/CIRCULATIONAHA.109.876185.

[13] V. S. Malik and F. B. Hu, "Sugar-Sweetened Beverages and Cardiometabolic Health: An Update of the Evidence," Nutrients, vol. 11, no. 8, p. 1840, Aug. 2019, doi: 10.3390/nu11081840.

[14] G. M. Singh, R. Micha, S. Khatibzadeh, S. Lim, M. Ezzati, and D. Mozaffarian, "Estimated Global, Regional, and National Disease Burdens Related to Sugar-Sweetened Beverage Consumption in 2010," Circulation, vol. 132, no. 8, pp. 639-666, Aug. 2015, doi: 10.1161/CIRCULATIONAHA.114.010636.

[15] A. Rosinger, K. Herrick, J. Gahche, and S. Park, "Sugar-sweetened Beverage Consumption Among U.S. Adults, 2011-2014," NCHS Data Brief, no. 270, pp. 1-8, Jan. 2017. 
[16] A. Rosinger, K. Herrick, J. Gahche, and S. Park, "Sugar-sweetened Beverage Consumption Among U.S. Youth, 2011-2014," NCHS Data Brief, no. 271, pp. 1-8, Jan. 2017.

[17] M. M. Lee, E. Altman, and K. A. Madsen, "Secular Trends in Sugar-Sweetened Beverage Consumption Among Adults, Teens, and Children: The California Health Interview Survey, 2011-2018," Prev. Chronic. Dis., vol. 18, p. 200399, Feb. 2021, doi: 10.5888/pcd18.200399.

[18] A. Chatelan et al., "Time trends in consumption of sugar-sweetened beverages and related socioeconomic differences among adolescents in Eastern Europe: signs of a nutrition transition?," Am. J. Clin. Nutr., p. nqab175, Jun. 2021, doi: 10.1093/ajcn/nqab175.

[19] S. B. O. Epifânio, J. A. C. da Silveira, R. C. E. de Menezes, P. M. Marinho, K. M. de M. Brebal, and G. Longo-Silva, "Análise de série temporal do consumo de bebidas açucaradas entre adultos no Brasil: 2007 a 2014," Ciênc. Saúde Coletiva, vol. 25, no. 7, pp. 2529-2540, Jul. 2020, doi: 10.1590/1413-81232020257.19402018.

[20] F. B. Hu, "Resolved: there is sufficient scientific evidence that decreasing sugar-sweetened beverage consumption will reduce the prevalence of obesity and obesity-related diseases: Sugar-sweetened beverages and risk of obesity," Obes. Rev., vol. 14, no. 8, pp. 606619, Aug. 2013, doi: 10.1111/obr.12040.

[21] D. Moher et al., "Preferred reporting items for systematic reviews and meta-analyses: The PRISMA statement," PLoS Med., vol. 6, no. 7, Jul. 2009, doi: 10.1371/journal.pmed.1000097.

[22] R. DerSimonian and N. Laird, "Meta-analysis in clinical trials," Control. Clin. Trials, vol. 7, no. 3, pp. 177-188, 1986, doi: 10.1016/0197-2456(86)90046-2.

[23] C. Willi, P. Bodenmann, W. A. Ghali, P. D. Faris, and J. Cornuz, "Active smoking and the risk of type 2 diabetes: A systematic review and meta-analysis," J. Am. Med. Assoc., vol. 298, no. 22, pp. 2654-2664, Dec. 2007, doi: 10.1001/jama.298.22.2654.

[24] J. Zhang and K. F. Yu, "What's the relative risk? A method of correcting the odds ratio in cohort studies of common outcomes," J. Am. Med. Assoc., vol. 280, no. 19, pp. 1690-1691, Nov. 1998, doi: 10.1001/jama.280.19.1690.

[25] J. P. T. Higgins and S. G. Thompson, "Quantifying heterogeneity in a meta-analysis," Stat. Med., vol. 21, no. 11, pp. 1539-1558, Jun. 2002, doi: $10.1002 / \operatorname{sim} .1186$.

[26] J. P. T. Higgins, "Measuring inconsistency in meta-analyses," BMJ, vol. 327, no. 7414, pp. 557-560, Sep. 2003, doi: 10.1136/bmj.327.7414.557.

[27] T. Lytras, “Web Application to Run Meta-Analyses [R package miniMeta version 0.2]," Mar. 01, 2020. https://cran.rproject.org/package=miniMeta (accessed Apr. 15, 2021).

[28] G. Wells, B. Shea, D. O'Connell, and J. Peterson, "The Newcastle-Ottawa Scale (NOS) for assessing the quality of nonrandomised studies in meta-analyses," Ottawa, ON: Ottawa Hospital Research Institute, 2000.

http://www.ohri.ca/programs/clinical_epidemiology/oxford.asp (accessed Nov. 10, 2019).

[29] M. Jawad, E. P. Vamos, M. Najim, B. Roberts, and C. Millett, "Impact of armed conflict on cardiovascular disease risk: a systematic review," Heart, vol. 105, no. 18, pp. 1388-1394, Sep. 2019, doi: 10.1136/heartjnl-2018-314459.

[30] R. H. Jenkins, E. P. Vamos, D. Taylor-Robinson, C. Millett, and A. A. Laverty, "Impacts of the 2008 Great Recession on dietary intake: a systematic review and meta-analysis," Int. J. Behav. Nutr. Phys. Act., vol. 18, no. 1, p. 57, Dec. 2021, doi: 10.1186/s12966-021-01125-8.

[31] M. Egger, G. D. Smith, M. Schneider, and C. Minder, "Bias in meta-analysis detected by a simple, graphical test," BMJ, vol. 315, no. 7109, pp. 629-634, Sep. 1997, doi: 10.1136/bmj.315.7109.629.

[32] J. Montonen, R. Järvinen, P. Knekt, M. Heliövaara, and A. Reunanen, “Consumption of Sweetened Beverages and Intakes of Fructose and Glucose Predict Type 2 Diabetes Occurrence," J. Nutr., vol. 137, no. 6, pp. 1447-1454, Jun. 2007, doi: 10.1093/jn/137.6.1447.

[33] J. R. Palmer, "Sugar-Sweetened Beverages and Incidence of Type 2 Diabetes Mellitus in African American Women," Arch. Intern. Med., vol. 168 , no. 14, p. 1487, Jul. 2008, doi: 10.1001/archinte.168.14.1487. 
[34] J. A. Nettleton, P. L. Lutsey, Y. Wang, J. A. Lima, E. D. Michos, and D. R. Jacobs, “Diet Soda Intake and Risk of Incident Metabolic Syndrome and Type 2 Diabetes in the Multi-Ethnic Study of Atherosclerosis (MESA)," Diabetes Care, vol. 32, no. 4, pp. 688-694, Apr. 2009, doi: $10.2337 /$ dc08-1799.

[35] L. de Koning, V. S. Malik, E. B. Rimm, W. C. Willett, and F. B. Hu, “Sugar-sweetened and artificially sweetened beverage consumption and risk of type 2 diabetes in men," Am. J. Clin. Nutr., vol. 93, no. 6, pp. 1321-1327, Jun. 2011, doi: 10.3945/ajen.110.007922.

[36] E. S. Eshak, H. Iso, T. Mizoue, M. Inoue, M. Noda, and S. Tsugane, "Soft drink, 100\% fruit juice, and vegetable juice intakes and risk of diabetes mellitus," Clin. Nutr., vol. 32, no. 2, pp. 300-308, Apr. 2013, doi: 10.1016/j.clnu.2012.08.003.

[37] G. Fagherazzi, A. Vilier, D. Saes Sartorelli, M. Lajous, B. Balkau, and F. Clavel-Chapelon, “Consumption of artificially and sugarsweetened beverages and incident type 2 diabetes in the Etude Epidémiologique auprès des femmes de la Mutuelle Générale de l'Education Nationale-European Prospective Investigation into Cancer and Nutrition cohort," Am. J. Clin. Nutr., vol. 97, no. 3, pp. 517-523, Mar. 2013, doi: 10.3945/ajcn.112.050997.

[38] L. O'Connor, F. Imamura, M. A. H. Lentjes, K.-T. Khaw, N. J. Wareham, and N. G. Forouhi, "Prospective associations and population impact of sweet beverage intake and type 2 diabetes, and effects of substitutions with alternative beverages," Diabetologia, vol. 58, no. 7 , pp. 1474-1483, Jul. 2015, doi: 10.1007/s00125-015-3572-1.

[39] K. Papier et al., "Consumption of sugar-sweetened beverages and type 2 diabetes incidence in Thai adults: results from an 8-year prospective study," Nutr. Diabetes, vol. 7, no. 6, pp. e283-e283, Jun. 2017, doi: 10.1038/nutd.2017.27.

[40] J.-P. Drouin-Chartier et al., "Changes in Consumption of Sugary Beverages and Artificially Sweetened Beverages and Subsequent Risk of Type 2 Diabetes: Results From Three Large Prospective U.S. Cohorts of Women and Men,” Diabetes Care, vol. 42, no. 12, pp. 21812189, Dec. 2019, doi: 10.2337/dc19-0734.

[41] K. M. Hirahatake et al., "Cumulative intake of artificially sweetened and sugar-sweetened beverages and risk of incident type 2 diabetes in young adults: the Coronary Artery Risk Development In Young Adults (CARDIA) Study," Am. J. Clin. Nutr., vol. 110, no. 3, pp. 733-741, Sep. 2019, doi: 10.1093/ajcn/nqz154.

[42] D. Stern et al., "Sugar-Sweetened Soda Consumption Increases Diabetes Risk Among Mexican Women,” J. Nutr., vol. 149, no. 5, pp. 795-803, May 2019, doi: 10.1093/jn/nxy298.

[43] A. O. Odegaard, W.-P. Koh, K. Arakawa, M. C. Yu, and M. A. Pereira, “Soft Drink and Juice Consumption and Risk of Physiciandiagnosed Incident Type 2 Diabetes: The Singapore Chinese Health Study," Am. J. Epidemiol., vol. 171, no. 6, pp. 701-708, Mar. 2010, doi: 10.1093/aje/kwp452.

[44] M. B. Schulze, “Sugar-Sweetened Beverages, Weight Gain, and Incidence of Type 2 Diabetes in Young and Middle-Aged Women," JAMA, vol. 292, no. 8, p. 927, Aug. 2004, doi: 10.1001/jama.292.8.927.

[45] R. Micha, J. L. Peñalvo, F. Cudhea, F. Imamura, C. D. Rehm, and D. Mozaffarian, "Association Between Dietary Factors and Mortality From Heart Disease, Stroke, and Type 2 Diabetes in the United States," JAMA, vol. 317, no. 9, p. 912, Mar. 2017, doi: 10.1001/jama.2017.0947.

[46] R. Dhingra et al., "Soft Drink Consumption and Risk of Developing Cardiometabolic Risk Factors and the Metabolic Syndrome in Middle-Aged Adults in the Community," Circulation, vol. 116, no. 5, pp. 480-488, Jul. 2007, doi: 10.1161/CIRCULATIONAHA.107.689935.

[47] S. Shin, S.-A. Kim, J. Ha, and K. Lim, "Sugar-Sweetened Beverage Consumption in Relation to Obesity and Metabolic Syndrome among Korean Adults: A Cross-Sectional Study from the 2012-2016 Korean National Health and Nutrition Examination Survey (KNHANES)," Nutrients, vol. 10, no. 10, p. 1467, Oct. 2018, doi: 10.3390/nu10101467.

[48] A. Garduño-Alanís et al., "Association between soft drink, fruit juice consumption and obesity in Eastern Europe: cross-sectional and longitudinal analysis of the HAPIEE study," J. Hum. Nutr. Diet., vol. 33, no. 1, pp. 66-77, Feb. 2020, doi: 10.1111/jhn.12696.

[49] D. A. Boggs, L. Rosenberg, P. F. Coogan, K. H. Makambi, L. L. Adams-Campbell, and J. R. Palmer, "Restaurant foods, sugar-sweetened soft drinks, and obesity risk among young African American women," Ethn. Dis., vol. 23, no. 4, pp. 445-451, 2013. 
[50] T. T. Fung, V. Malik, K. M. Rexrode, J. E. Manson, W. C. Willett, and F. B. Hu, "Sweetened beverage consumption and risk of coronary heart disease in women," Am. J. Clin. Nutr., vol. 89, no. 4, pp. 1037-1042, Apr. 2009, doi: 10.3945/ajcn.2008.27140.

[51] L. de Koning, V. S. Malik, M. D. Kellogg, E. B. Rimm, W. C. Willett, and F. B. Hu, "Sweetened Beverage Consumption, Incident Coronary Heart Disease, and Biomarkers of Risk in Men," Circulation, vol. 125, no. 14, pp. 1735-1741, Apr. 2012, doi:

10.1161/CIRCULATIONAHA.111.067017.

[52] A. Keller et al., "Substitution of sugar-sweetened beverages for other beverages and the risk of developing coronary heart disease: Results from the Harvard Pooling Project of Diet and Coronary Disease," Prev. Med., vol. 131, p. 105970, Feb. 2020, doi: 10.1016/j.ypmed.2019.105970.

[53] E. S. Eshak et al., "Soft drink intake in relation to incident ischemic heart disease, stroke, and stroke subtypes in Japanese men and women: the Japan Public Health Centre-based study cohort l," Am. J. Clin. Nutr., vol. 96, no. 6, pp. 1390-1397, Dec. 2012, doi:

10.3945/ajcn.112.037903.

[54] S. C. Larsson, A. Åkesson, and A. Wolk, "Sweetened Beverage Consumption Is Associated with Increased Risk of Stroke in Women and Men," J. Nutr., vol. 144, no. 6, pp. 856-860, Jun. 2014, doi: 10.3945/jn.114.190546.

[55] M. P. Pase et al., "Sugar- and Artificially Sweetened Beverages and the Risks of Incident Stroke and Dementia: A Prospective Cohort Study," Stroke, vol. 48, no. 5, pp. 1139-1146, May 2017, doi: 10.1161/STROKEAHA.116.016027.

[56] L. S. Pacheco et al., "Sugar-Sweetened Beverage Intake and Cardiovascular Disease Risk in the California Teachers Study," J. Am. Heart Assoc., vol. 9, no. 10, May 2020, doi: 10.1161/JAHA.119.014883.

[57] A. M. Bernstein, L. de Koning, A. J. Flint, K. M. Rexrode, and W. C. Willett, "Soda consumption and the risk of stroke in men and women,” Am. J. Clin. Nutr., vol. 95, no. 5, pp. 1190-1199, May 2012, doi: 10.3945/ajcn.111.030205.

[58] F. Song, K. S. Khan, J. Dinnes, and A. J. Sutton, "Asymmetric funnel plots and publication bias in meta-analyses of diagnostic accuracy," Int. J. Epidemiol., vol. 31, no. 1, pp. 88-95, Feb. 2002, doi: 10.1093/ije/31.1.88.

[59] D. C. Greenwood et al., "Association between sugar-sweetened and artificially sweetened soft drinks and type 2 diabetes: systematic review and dose-response meta-analysis of prospective studies," Br. J. Nutr., vol. 112, no. 5, pp. 725-734, Sep. 2014, doi: $10.1017 /$ S0007114514001329.

[60] V. S. Malik, A. Pan, W. C. Willett, and F. B. Hu, "Sugar-sweetened beverages and weight gain in children and adults: a systematic review and meta-analysis," Am. J. Clin. Nutr., vol. 98, no. 4, pp. 1084-1102, Oct. 2013, doi: 10.3945/ajcn.113.058362.

[61] A. Narain, C. S. Kwok, and M. A. Mamas, "Soft drinks and sweetened beverages and the risk of cardiovascular disease and mortality: a systematic review and meta-analysis," Int. J. Clin. Pract., vol. 70, no. 10, pp. 791-805, Oct. 2016, doi: 10.1111/ijcp.12841.

[62] C. Huang, J. Huang, Y. Tian, X. Yang, and D. Gu, "Sugar sweetened beverages consumption and risk of coronary heart disease: A meta-analysis of prospective studies," Atherosclerosis, vol. 234, no. 1, pp. 11-16, May 2014, doi: 10.1016/j.atherosclerosis.2014.01.037.

[63] S. L. Tey, N. B. Salleh, J. Henry, and C. G. Forde, "Effects of aspartame-, monk fruit-, stevia- and sucrose-sweetened beverages on postprandial glucose, insulin and energy intake," Int. J. Obes., vol. 41, no. 3, pp. 450-457, Mar. 2017, doi: 10.1038/ijo.2016.225.

[64] L. Solomi, G. A. Rees, and K. M. Redfern, "The acute effects of the non-nutritive sweeteners aspartame and acesulfame-K in UK diet cola on glycaemic response," Int. J. Food Sci. Nutr., vol. 70, no. 7, pp. 894-900, Oct. 2019, doi: 10.1080/09637486.2019.1585418.

[65] G. Livesey et al., "Dietary Glycemic Index and Load and the Risk of Type 2 Diabetes: A Systematic Review and Updated MetaAnalyses of Prospective Cohort Studies," Nutrients, vol. 11, no. 6, p. 1280, Jun. 2019, doi: 10.3390/nu11061280.

[66] A. Naska, A. Lagiou, and P. Lagiou, "Dietary assessment methods in epidemiological research: current state of the art and future prospects," F1000Research, vol. 6, p. 926, Jun. 2017, doi: 10.12688/f1000research.10703.1.

[67] F. Riordan et al., "A systematic review of methods to assess intake of sugar-sweetened beverages among healthy European adults and children: a DEDIPAC (DEterminants of Dlet and Physical Activity) study," Public Health Nutr., vol. 20, no. 4, pp. 578-597, Mar. 2017, doi: $10.1017 /$ S1368980016002639.

Page 23/26 
[68] M. Ezzati, Z. Obermeyer, I. Tzoulaki, B. M. Mayosi, P. Elliott, and D. A. Leon, "Contributions of risk factors and medical care to cardiovascular mortality trends," Nat. Rev. Cardiol., vol. 12, no. 9, pp. 508-530, Sep. 2015, doi: 10.1038/nrcardio.2015.82.

[69] G. Rose, "Incubation period of coronary heart disease," Int. J. Epidemiol., vol. 34, no. 2, pp. 242-244, Apr. 2005, doi:

10.1093/ije/dyh308.

[70] L. T. Budnik et al., "Diagnosis, monitoring and prevention of exposure-related non-communicable diseases in the living and working environment: DiMoPEx-project is designed to determine the impacts of environmental exposure on human health," J. Occup. Med.

Toxicol., vol. 13, no. 1, p. 6, Dec. 2018, doi: 10.1186/s12995-018-0186-9.

[71] Z. Fewell, G. Davey Smith, and J. A. C. Sterne, "The Impact of Residual and Unmeasured Confounding in Epidemiologic Studies: A Simulation Study," Am. J. Epidemiol., vol. 166, no. 6, pp. 646-655, Jun. 2007, doi: 10.1093/aje/kwm165.

[72] A. Paez, "Gray literature: An important resource in systematic reviews: PAEZ," J. Evid.-Based Med., vol. 10, no. 3, pp. 233-240, Aug. 2017, doi: 10.1111/jebm.12266.

\section{Figures}

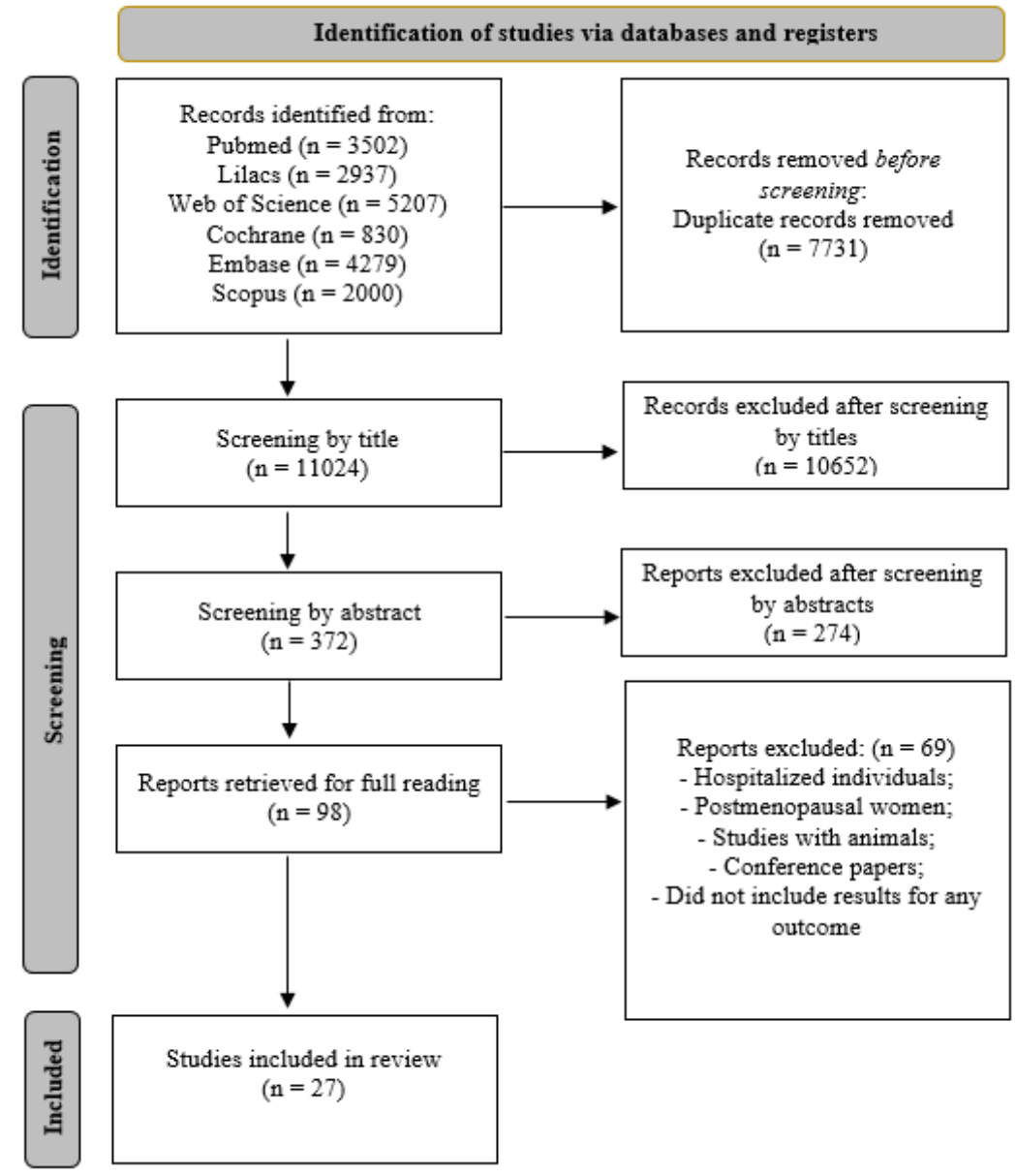

Figure 1

Flow diagram of articles selection stages. 
Obesity

Study

Risk Ratio

RR

95\%-Cl Weight

Low/Moderate intake

Dhingra et al., 2007

Boggs et al., 2013

Shin et al., 2018

Garduno-Alanis et al., 2020

Random-effects model

Heterogeneity: $I^{2}=36 \%, p=0.20$
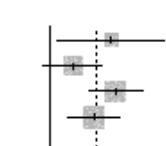

$1.23(1.02-1.48) \quad 11.0 \%$ $1.08(0.98-1.20) \quad 26.9 \%$

$1.25(1.14-1.37) \quad 29.9 \%$

$1.16(1.06-1.26) \quad 32.3 \%$

$1.17(1.10-1.25) 100.0 \%$
Study
High intake
Dhingra et al., 2007
Boggs et al., 2013
Random-effects model
Heterogeneity: $I^{2}=30 \%, p=0.23$
RR 95\%-Cl Weight
Risk Ratio
$1.29(1.05-1.58) \quad 31.3 \%$
$1.12(1.00-1.25) \quad 68.7 \%$
$1.17(1.03-1.33) 100.0 \%$
0.5

12

1

(20)

Diabetes

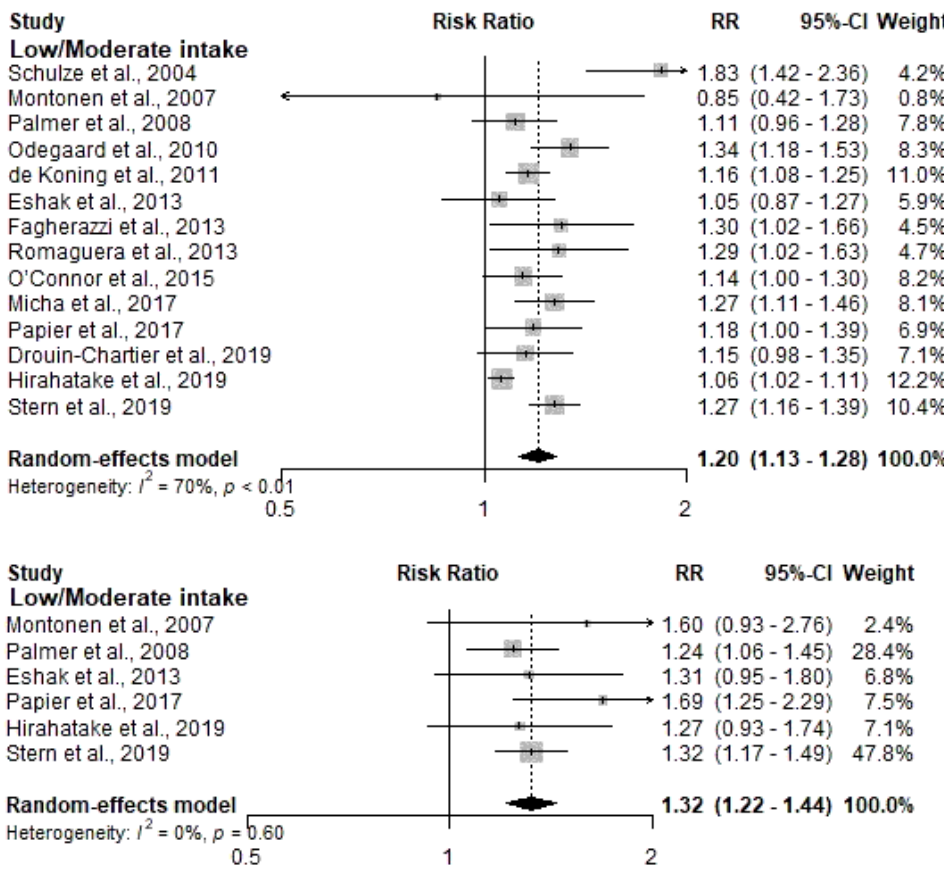

\section{Figure 2}

Pooled effect for the association between sugar sweetened beverages intake and obesity and type 2 diabetes among adults. 


\section{Coronary heart disease}

Study

Risk Ratio

RR

95\%-Cl Weight

Fung et al., 2009

de Koning et al., 2012

Keller et al., 2020

Random-effects mode

Heterogeneity: $l^{2}=66 \%, p=0.06$

0.5

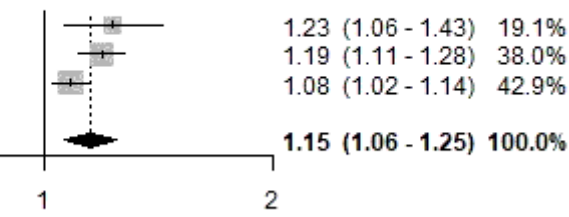

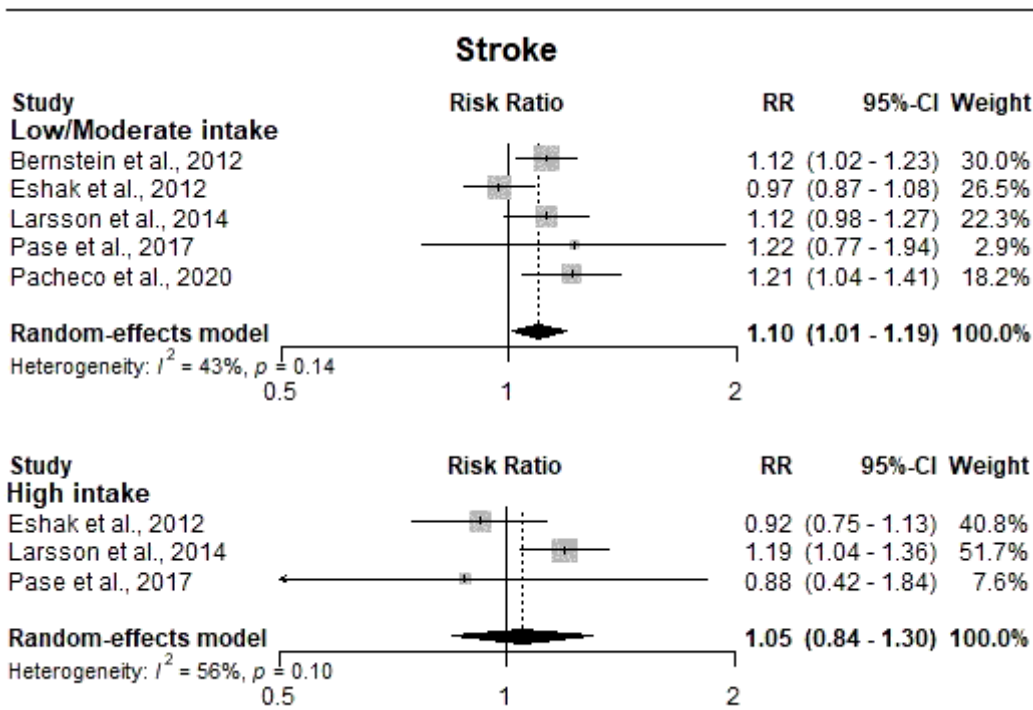

Figure 3

Pooled effect for the association between sugar sweetened beverages intake and coronary heart disease and stroke among adults.

\section{Supplementary Files}

This is a list of supplementary files associated with this preprint. Click to download.

- Supplementaryinformation.docx 